\title{
Search for New Physics with Same-Sign Isolated Dilepton Events with Jets and Missing Transverse Energy
}

\author{
S. Chatrchyan et al. ${ }^{*}$ \\ (CMS Collaboration)
}

(Received 30 May 2012; published 16 August 2012)

\begin{abstract}
A search for new physics is performed in events with two same-sign isolated leptons, hadronic jets, and missing transverse energy in the final state. The analysis is based on a data sample corresponding to an integrated luminosity of $4.98 \mathrm{fb}^{-1}$ produced in $p p$ collisions at a center-of-mass energy of $7 \mathrm{TeV}$ collected by the CMS experiment at the LHC. This constitutes a factor of 140 increase in integrated luminosity over previously published results. The observed yields agree with the standard model predictions and thus no evidence for new physics is found. The observations are used to set upper limits on possible new physics contributions and to constrain supersymmetric models. To facilitate the interpretation of the data in a broader range of new physics scenarios, information on the event selection, detector response, and efficiencies is provided.
\end{abstract}

DOI: 10.1103/PhysRevLett.109.071803

PACS numbers: 12.60.Jv, 13.85.Rm, 14.80.Ly

The standard model (SM) is a very successful theory of elementary particles and their interactions. It is generally believed that new physics (NP) could manifest itself at the $\mathrm{TeV}$ scale. Supersymmetry (SUSY) is one of these attractive possibilities. It leads to gauge coupling unification at very high energy, provides a mechanism to mitigate large radiative corrections to the Higgs mass and, in its $R$-parityconserving [1] realization, can provide a dark matter candidate. A comprehensive program of searches for the production of supersymmetric particles has been underway since 2010 at the Large Hadron Collider (LHC). Since SUSY models vary widely, these searches target a broad range of possible final states, including purely hadronic states [2,3], leptonic states with one lepton [4,5], two leptons of the opposite sign [6,7], two leptons of the same sign [6,8], and three or more leptons [9], as well as photonic final states $[10,11]$.

In this Letter we report on a search for NP based on isolated same-sign (SS) dileptons, missing transverse energy $\left(E_{\mathrm{T}}^{\text {miss }}\right)$, and hadronic jets. In SUSY SS dileptons can arise, for example, from pair production of colored superpartners (gluinos and/or squarks), with a lepton in the decay chain of each primary SUSY particle [12-14]; more generally, this signature is sensitive to final states with same-sign $W$ bosons and/or top quarks [15-20]. The rarity of SS dileptons in the SM makes a NP search in this final state particularly attractive.

All types of charged leptons, $e, \mu$, and hadronically decaying $\tau \mathrm{s}$, are included in our search. These final states

*Full author list given at the end of the article.

Published by the American Physical Society under the terms of the Creative Commons Attribution 3.0 License. Further distribution of this work must maintain attribution to the author(s) and the published article's title, journal citation, and DOI. are indicators of the possible presence of SUSY particles as well as other possible NP scenarios. The results are based on a data sample corresponding to $4.98 \pm 0.11 \mathrm{fb}^{-1}$ of $p p$ collisions at a center-of-mass energy of $7 \mathrm{TeV}$ collected in 2011 by the Compact Muon Solenoid (CMS) [21] experiment at the LHC. This study results in a major improvement in sensitivity with respect to the search performed with data collected in 2010 [8] because of the 140-fold increase in the integrated luminosity of the data sample. These results are interpreted using the constrained minimal supersymmetric extension of the standard model (CMSSM) [22]. In addition, this analysis provides information on the event selection and detector response in order to facilitate the application of our results to a broader range of NP scenarios.

A detailed description of the CMS detector is found elsewhere [21]. Its central feature is a superconducting solenoid providing an axial magnetic field of $3.8 \mathrm{~T}$. Muons are measured in gas detectors embedded in the steel return yoke of the magnet, while all other particle detection systems are located inside the bore of the solenoid. Charged particle trajectories are measured by a silicon pixel and strip tracker system, covering $|\eta|<2.5$, where the pseudorapidity is defined as $\eta=-\ln [\tan \theta / 2]$, and $\theta$ is the polar angle with respect to the counterclockwise beam direction. A crystal electromagnetic calorimeter (ECAL) and a brass-scintillator hadronic calorimeter surround the tracker volume. In addition, the CMS detector has an extensive forward calorimeter and nearly hermetic $4 \pi$ coverage. The CMS trigger consists of a two-stage system. The first level of the CMS trigger system, composed of custom hardware processors, uses information from the calorimeters and muon detectors to select a subset of the events. The high level trigger processor farm further decreases the event rate from around $100 \mathrm{kHz}$ to around $300 \mathrm{~Hz}$, before data storage. 
All lepton candidates are required to have $|\eta|<2.4$ and to be consistent with a common interaction vertex. Muon candidates are reconstructed [23] by matching tracks in the silicon detector to signals in the muon system. The reconstruction of muons is refined further by imposing track quality and calorimeter energy deposition requirements. Electron candidates are reconstructed [24] starting from a cluster of energy deposits in the ECAL, which is then matched to signals in the silicon tracker. The energy shower in the ECAL must have a shape consistent with expectations for electron showers and its position is required to be well matched to the extrapolated track. Both electrons and muons are required to be isolated from other activity in the event. This is achieved using a scalar sum of transverse track momenta and transverse calorimeter energy deposits, within $\Delta R \equiv \sqrt{(\Delta \phi)^{2}+(\Delta \eta)^{2}}<0.3$ of the candidate's direction, where $\phi$ is the azimuthal angle. The sum is required to be less than $15 \%$ of the candidate's transverse momentum $\left(p_{\mathrm{T}}\right)$. Hadronic $\tau$ candidates are reconstructed using the Hadron plus Strip algorithm [25]. We select isolated hadronic $\tau$ candidates with one or three charged hadrons in a narrow cone around the $\tau$ direction.

Jets and $E_{\mathrm{T}}^{\text {miss }}$ are reconstructed using the particle-flow technique [26,27]. For jet clustering, the anti- $k_{\mathrm{T}}$ algorithm is used with the distance parameter $R=0.5$ [28]. We require selected jets to have $p_{\mathrm{T}}>40 \mathrm{GeV}$ and $|\eta|<2.5$ to be considered for analysis. The $H_{\mathrm{T}}$ is defined to be the scalar sum of the $p_{\mathrm{T}}$ of all selected jets whose angular distance to the nearest lepton satisfies $\Delta R>0.4$. Events are required to have two same-sign leptons and at least two jets. A minimum dilepton invariant mass of $8 \mathrm{GeV}$ is required in order to suppress the low-mass dilepton background. Events having a third lepton are removed if two of the leptons form a $Z$ boson candidate with an invariant mass within $\pm 15 \mathrm{GeV}$ of the $Z$ boson mass.

Three selection strategies are followed to maximize the sensitivity to the presence of NP. The first one is to use a fully efficient dilepton and $H_{\mathrm{T}}$ based trigger in the $e e, \mu \mu$, and $e \mu$ channels with $p_{\mathrm{T}}^{\mu}>5 \mathrm{GeV}$ and $p_{\mathrm{T}}^{e}>10 \mathrm{GeV}$, and a requirement of $H_{\mathrm{T}}>200 \mathrm{GeV}$ applied to the offline reconstructed objects. The second strategy trades an increased lepton $p_{\mathrm{T}}$ threshold against a reduced $H_{\mathrm{T}}$ threshold. Here both leptons are required to have $p_{\mathrm{T}}>10 \mathrm{GeV}$ and at least one to have $p_{\mathrm{T}}>20 \mathrm{Gev}$. Such events are collected with a purely leptonic trigger with no requirement on $H_{\mathrm{T}}$. The third strategy focuses on $\tau e, \tau \mu$ and $\tau \tau$ final states with $p_{\mathrm{T}}^{\mu}>$ $5 \mathrm{GeV}, p_{\mathrm{T}}^{e}>10 \mathrm{GeV}$, and $p_{\mathrm{T}}^{\tau}>15 \mathrm{GeV}$. Triggers for hadronic $\tau$-leptons typically lead to high rates. For this reason dedicated triggers are used that rely on significant $H_{\mathrm{T}}$ and $E_{\mathrm{T}}^{\text {miss }}$, in addition to the presence of a single lepton or two hadronic $\tau$-leptons.

Using $R$-parity-conserving SUSY as a guiding example, we note that the simplest incarnation of the topology probed by this analysis involves three distinct mass scales. In this example, these masses would belong to the gluino, chargino, and lightest SUSY particle (LSP). The mass differences of these particles can strongly influence the kinematics of the final-state objects, hence affecting several main observables used in this analysis: lepton $p_{\mathrm{T}}, H_{\mathrm{T}}$, and $E_{\mathrm{T}}^{\mathrm{miss}}$. Therefore, in order to maximize the sensitivity of our analysis to a variety of NP scenarios, we define multiple search regions in the $\left(H_{\mathrm{T}}, E_{\mathrm{T}}^{\text {miss }}\right)$ plane : Region 1 $\left(H_{\mathrm{T}}>80 \mathrm{GeV}, \quad E_{\mathrm{T}}^{\text {miss }}>120 \mathrm{GeV}\right)$, Region $2\left(H_{\mathrm{T}}>\right.$ $\left.200 \mathrm{GeV}, E_{\mathrm{T}}^{\text {miss }}>120 \mathrm{GeV}\right)$, Region $3\left(H_{\mathrm{T}}>450 \mathrm{GeV}\right.$, $\left.E_{\mathrm{T}}^{\text {miss }}>50 \mathrm{GeV}\right)$, Region $4\left(H_{\mathrm{T}}>450 \mathrm{GeV}, E_{\mathrm{T}}^{\text {miss }}>\right.$ $120 \mathrm{GeV})$, and Region $5\left(H_{\mathrm{T}}>450 \mathrm{GeV}, E_{\mathrm{T}}^{\text {miss }}>0 \mathrm{GeV}\right)$. The $H_{\mathrm{T}}$ requirements of $200 \mathrm{GeV}$ and $450 \mathrm{GeV}$ are also motivated in part by trigger thresholds. A scatter plot of events observed in these search regions is shown in Fig. 1.

The background for the same-sign dilepton topology has three components: irreducible background from rare SM processes; leptons resulting from semileptonic decays within a jet, or jets mimicking leptons in events with zero or one genuine isolated lepton; and opposite-sign dilepton events where the charge of one of the two leptons has been mismeasured.

The irreducible backgrounds are dominated by $t \bar{t}+$ $W^{ \pm} / Z, W^{ \pm} W^{ \pm} q q$, and $W^{ \pm} Z$ production, combining in similar parts to about $95 \%$ of the total. The remaining contributions originate from processes such as triboson and $Z Z$ production, $W^{ \pm} Z+\gamma$, and double-parton scattering $2 \times\left(q \bar{q}^{\prime} \rightarrow W^{ \pm}\right)$, in decending order of importance. All irreducible backgrounds are estimated using leading-order Monte Carlo simulation normalized to the next-to-leading-order (NLO) production cross sections. Events are generated with the MADGRAPH [29] event generator and then passed on to PYTHIA [30] for parton shower

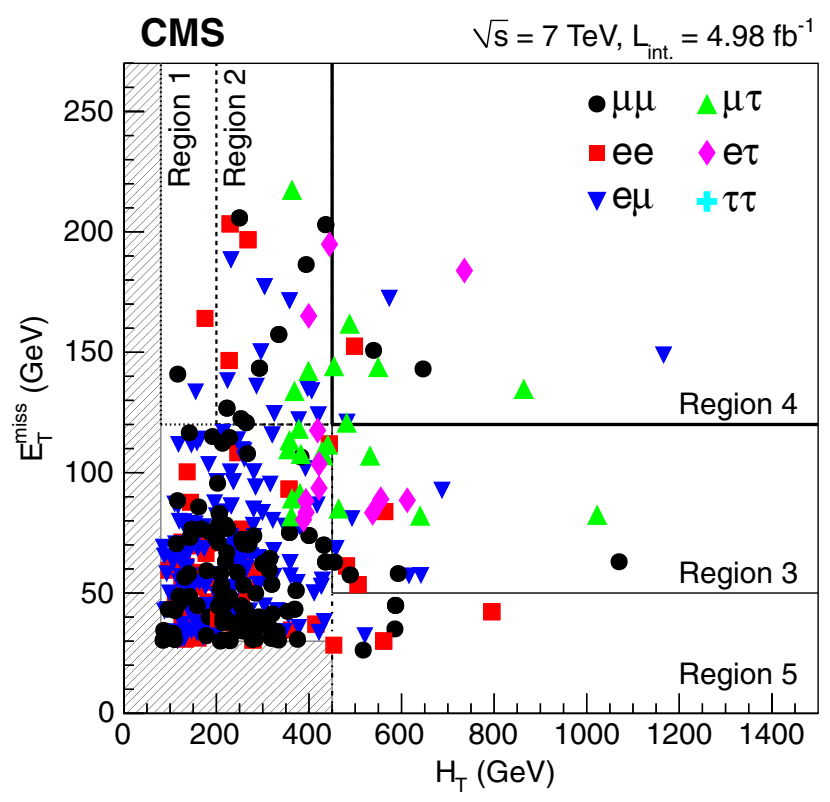

FIG. 1 (color online). Selected SS dilepton events in the various search regions displayed in the $H_{\mathrm{T}}, E_{\mathrm{T}}^{\text {miss }}$ plane. 
and hadronization. The generated events are processed by the CMS event simulation and the same chain of reconstruction programs used for collision data. A 50\% systematic uncertainty is assigned to this irreducible background prediction. These processes constitute $35 \%-75 \%$ of the total background, depending on the search region.

The background due to lepton candidates originating from jets, hereafter referred as nonprompt, forms $20 \%-60 \%$ of the total background. Such candidates can be genuine leptons, for example, from heavy-flavor decays, hadrons reconstructed as leptons, or jets fluctuating to give hadronic $\tau$ signatures. We have developed and validated a set of techniques to measure this background from data. In each case, a tag-and-probe method is applied to a control sample rich in two-jet events containing leptons selected with loose requirements to measure the conditional probability that the probe jet yields a candidate passing tight lepton requirements. This probability, measured as a function of jet kinematics and event characteristics, is then applied to signal sidebands to estimate nonprompt lepton backgrounds. This suite of techniques encompasses a range of control samples, jet tags, lepton requirements, and variations in the jet kinematics to provide independent and complementary assessments of 50\% systematic uncertainties. Full details are given in Ref. [8]. At least two techniques are used in all non- $\tau$ dilepton modes and they yield consistent results within their respective uncertainties.

We quantify backgrounds from events with lepton charge misreconstruction by analyzing SS $e e$ or $\tau \tau$ events inside the $Z$ mass peak [8]. This background forms less than $5 \%$ of the total background across all search regions. The charge misreconstruction probability for muons is of the order of $10^{-5}$ and can be neglected.

We determine the performance of the background prediction methods using the low $H_{\mathrm{T}}$ and low $E_{\mathrm{T}}^{\text {miss }}$ region in the data that is expected to be dominated by SM events. We find good agreement between observed yields and the predicted background.

We show the predicted background contributions from each source mentioned above as well as the observed event yields in Fig. 2 and summarize them in Table I for each search region. The beam related multiple interactions do not alter these results. There is no evidence of an excess over the expected SM predictions. This measurement is used together with the uncertainty on the signal acceptance to set an upper limit (UL) on the contribution from NP events.

We measure the electron and muon selection efficiencies in data and simulation using $Z$ events to derive simulationto-data correction factors. The uncertainty on the combined lepton selection efficiency decreases with lepton $p_{\mathrm{T}}$, from $5 \%$ at the lowest $p_{\mathrm{T}}$ to $3 \%$ above $20 \mathrm{GeV}$. We assign an additional $5 \%$ systematic uncertainty per lepton to cover potential mismodeling of the lepton isolation efficiency due to varying hadronic activity in signal events. We estimate in

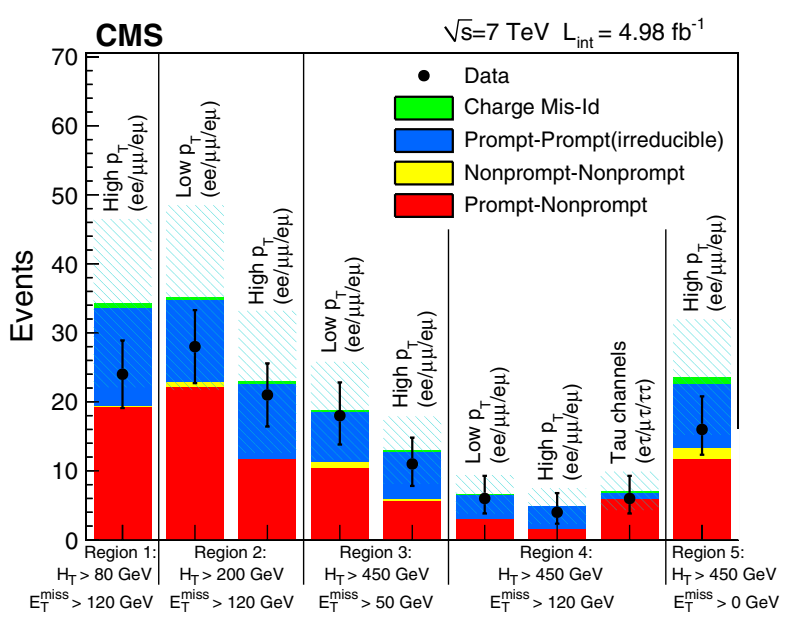

FIG. 2 (color online). Summary of background predictions and observed yields in the various search regions. Leptons from decays of $W, Z$, and NP particles are referred to as prompt leptons. The hatched bands represent the total uncertainty on the background predictions.

a sample of $Z \rightarrow \tau \tau$ events the uncertainty on the $\tau$ selection and reconstruction efficiency to be $10 \%$ [25].

We conservatively choose to attribute a flat uncertainty of $7.5 \%$ to the energy measurement of all jets as well as to the hadronic component used for the $E_{\mathrm{T}}^{\text {miss }}$ observable. The cumulative effect of this uncertainty on the signal acceptance is intrinsically model dependent. We observe uncertainties below $3 \%$ for models with characteristically high $H_{\mathrm{T}}$ scales, well above the $H_{\mathrm{T}}$ requirements. For models with characteristic $H_{\mathrm{T}}$ scales near or below the $H_{\mathrm{T}}$ requirements, uncertainties due to jet energy calibration can be as high as $30 \%$.

The theoretical uncertainties on the signal acceptance due to the modeling of initial- and final-state radiation and knowledge of the parton distribution functions are estimated to be $2 \%$. Using the LM6 benchmark model (CMSSM point with $m_{0}=85 \mathrm{GeV}, m_{1 / 2}=400 \mathrm{GeV}$, $\tan \beta=10, A_{0}=0$, and $\mu>0$ ) [31] as a signal model, the total experimental and theoretical uncertainties in the signal yield add up to $14 \%$ or $20 \%$ depending on the search region. This includes a $2.2 \%$ systematic uncertainty in the integrated luminosity [32].

We set a 95\% confidence level (CL) upper limits on the number of observed events using the modified frequentist construction $\mathrm{CL}_{s}$ method [33-35]. We assume log-normal distributions for the efficiency and background uncertainties. As a reference, we provide in Table I the upper limits based on a $20 \%$ signal acceptance uncertainty.

In order to compare our signal sensitivity to that of other searches for SUSY, we interpret the results in the context of the CMSSM model. We compare the observed upper limits on the number of signal events reported in Table I to the expected number of events in each signal region in the CMSSM model in a plane of $\left(m_{0}, m_{1 / 2}\right)$ for $\tan \beta=10$, 
TABLE I. Observed number of events in data compared to the predicted background yields for the considered search regions. The uncertainties include the statistical and systematic components added in quadrature with correlations taken into account. The 95\% CL upper limit (UL) on the contribution from NP events is also given.

\begin{tabular}{|c|c|c|c|c|c|}
\hline Region & \multicolumn{3}{|c|}{ Mode or $p_{\mathrm{T}}$ threshold } & Total & $\mathrm{UL}$ \\
\hline & \multicolumn{3}{|c|}{ High $p_{T}: p_{T}^{\ell 1, \ell 2}>20,10 \mathrm{GeV}$} & & \\
\hline & $e e$ & $\mu \mu$ & $e \mu$ & & \\
\hline \multirow[t]{2}{*}{1} & $6.8 \pm 2.7$ & $8.6 \pm 3.3$ & $18.7 \pm 6.9$ & $34.1 \pm 12.2$ & \\
\hline & 5 & 7 & 12 & 24 & 13.7 \\
\hline \multirow[t]{2}{*}{2} & $4.3 \pm 1.9$ & $6.1 \pm 2.4$ & $12.2 \pm 4.6$ & $22.6 \pm 8.3$ & \\
\hline & 4 & 6 & 11 & 21 & 15.1 \\
\hline \multirow[t]{2}{*}{3} & $3.8 \pm 1.7$ & $3.1 \pm 1.4$ & $6.1 \pm 2.4$ & $13.0 \pm 4.9$ & \\
\hline & 4 & 2 & 5 & 11 & 9.6 \\
\hline \multirow[t]{2}{*}{4} & $1.1 \pm 1.1$ & $1.2 \pm 1.2$ & $2.6 \pm 1.4$ & $4.9 \pm 2.6$ & \\
\hline & 1 & 0 & 3 & 4 & 6.2 \\
\hline \multirow[t]{4}{*}{5} & $9.1 \pm 3.6$ & $4.7 \pm 1.9$ & $9.8 \pm 3.7$ & $23.6 \pm 8.4$ & \\
\hline & 7 & 4 & 5 & 16 & 10.4 \\
\hline & \multicolumn{3}{|c|}{ Low $p_{T}: p_{T}^{e, \mu}>10,5 \mathrm{GeV}$} & & \\
\hline & $e e$ & $\mu \mu$ & $e \mu$ & & \\
\hline \multirow[t]{2}{*}{2} & $4.4 \pm 1.8$ & $14.1 \pm 6.0$ & $16.5 \pm 6.4$ & $35.0 \pm 13.4$ & \\
\hline & 4 & 10 & 14 & 28 & 16.9 \\
\hline \multirow[t]{2}{*}{3} & $3.4 \pm 1.6$ & $6.5 \pm 2.8$ & $8.9 \pm 3.6$ & $18.8 \pm 7.1$ & \\
\hline & 4 & 6 & 8 & 18 & 14.0 \\
\hline \multirow[t]{4}{*}{4} & $1.0 \pm 0.8$ & $2.4 \pm 1.2$ & $3.2 \pm 1.5$ & $6.6 \pm 2.8$ & \\
\hline & 1 & 2 & 3 & 6 & 7.4 \\
\hline & Tau & $\mathrm{s}: p_{T}^{e, \mu, \tau}>1$ & $\mathrm{GeV}$ & & \\
\hline & $e \tau$ & $\mu \tau$ & $\tau \tau$ & & \\
\hline \multirow[t]{2}{*}{4} & $2.6 \pm 1.0$ & $4.4 \pm 2.2$ & $0.0 \pm 0.1$ & $7.1 \pm 2.8$ & \\
\hline & 1 & 5 & 0 & 6 & 7.1 \\
\hline
\end{tabular}

$A_{0}=0$, and $\mu>0$. For each point in the CMSSM, we choose the signal region providing the best expected upper limit on the cross section to evaluate the observed limit; in all cases the best limit is achieved in Region 4, where high $p_{\mathrm{T}}$ leptons, large $H_{\mathrm{T}}>450 \mathrm{GeV}$, and $E_{\mathrm{T}}^{\text {miss }}>120 \mathrm{GeV}$ are required. We interpret all points having mean expected values above the corresponding observed upper limit as excluded at the $95 \%$ CL. For this exercise the systematic uncertainty on the signal acceptance is re-evaluated for each point in order for the upper limit to reflect the varying influences of the jet energy scale uncertainty. We display the observed exclusion region in Fig. 3. For $m_{0}>1.3 \mathrm{TeV}$, the exclusion curve flattens out at about $m_{1 / 2} \sim 290 \mathrm{GeV}$, which corresponds to a winolike $\tilde{\chi}_{1}^{ \pm}$mass of $\sim 200 \mathrm{GeV}$. The new result extends the excluded CMSSM region to gluino masses of $710 \mathrm{GeV}$. This exclusion includes a $-1 \sigma_{\text {th }}$ reduction to account for theory uncertainty [36-44] on the cross section; the limit is independent of the squark masses.

One of the challenges of signature-based searches is to convey information in a form that can be used to test a variety of NP models. In Ref. [8], additional information is presented that can be used to confront NP models in an approximate way through generator-level simulation studies. The approximate model of lepton, jet, and $E_{\mathrm{T}}^{\text {miss }}$ selection efficiencies in terms of the generator-level quantities are shown to be sufficiently accurate to reproduce the constraints on NP models that otherwise would require the full CMS detector simulation. The efficiency dependence can be parameterized as a function of $p_{\mathrm{T}}$ (expressed in $\mathrm{GeV})$ as $0.72\left\{\operatorname{erf}\left[\left(p_{\mathrm{T}}-10\right) / 22.5\right]\right\}+0.22\left\{1-\operatorname{erf}\left[\left(p_{\mathrm{T}}-\right.\right.\right.$ 10) $/ 22.5]\}$ for electrons, $0.79\left\{\operatorname{erf}\left[\left(p_{\mathrm{T}}-5\right) / 19.5\right]\right\}+$ $0.41\left\{1-\operatorname{erf}\left[\left(p_{\mathrm{T}}-5\right) / 19.5\right]\right\}$ for muons, and $0.341-$ $\exp \left[-0.052\left(p_{\mathrm{T}}-15\right)\right]$ for taus, where erf is the error function. We studied the efficiency for an event to pass a given reconstructed $E_{\mathrm{T}}^{\text {miss }}\left(H_{\mathrm{T}}\right)$ threshold as a function of the generator-level $E_{\mathrm{T}}^{\text {miss }}\left(H_{\mathrm{T}}\right)$, where in the latter case $E_{\mathrm{T}}^{\text {miss }}$ is computed using neutrinos and the LSPs and $H_{\mathrm{T}}$ is the scalar sum of the transverse momenta of the partons that satisfy the same jet selection criteria used in this analysis. The dependences are parameterized by $0.5 \epsilon_{\infty}\left\{\operatorname{erf}\left[\left(x-x_{1 / 2}\right) / \sigma\right]+1\right\}$, where $x$ corresponds to the generator-level $E_{\mathrm{T}}^{\text {miss }}$ or $H_{\mathrm{T}}, \epsilon_{\infty}$ is the selection efficiency plateau at high values of $x, x_{1 / 2}$ is the value of $x$ corresponding to half the plateau efficiency, and $\sigma$ determines how fast the efficiency changes with $x$. For the $H_{\mathrm{T}}$ selections of 200 and $450 \mathrm{GeV}$, the values of $\left(\epsilon_{\infty}, x_{1 / 2}, \sigma\right)$ are $(0.997,185 \mathrm{GeV}, 99 \mathrm{GeV})$, and $(0.992,441 \mathrm{GeV}, 120 \mathrm{GeV})$, respectively. For the $E_{\mathrm{T}}^{\text {miss }}$ selections of 50 and $120 \mathrm{GeV}$, the parameters are $(0.999,43 \mathrm{GeV}, 39 \mathrm{GeV})$, and $(0.999$, 


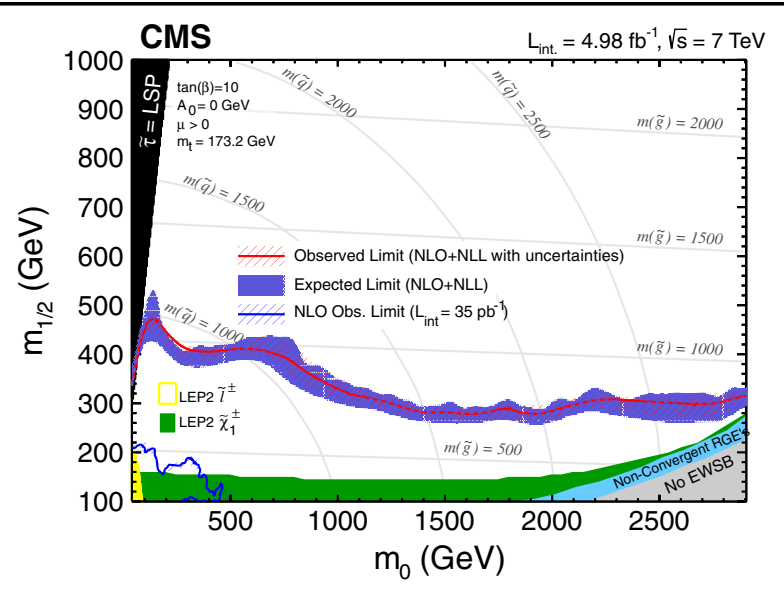

FIG. 3 (color online). Exclusion region, below the red curve, in the CMSSM corresponds to the observed upper limits on the number of events from NP. The central observed curve, which includes experimental uncertainties, is obtained using high $p_{\mathrm{T}}$ leptons with $H_{\mathrm{T}}>450 \mathrm{GeV}$ and $E_{\mathrm{T}}^{\text {miss }}>120 \mathrm{GeV}$. The hatched region corresponds to the theoretical uncertainties on the cross section, whereas the shaded region shows the experimental errors with $\pm 1 \sigma$ variation. We also show the result of the previous analysis [8] to illustrate the improvement.

$123 \mathrm{GeV}, 37 \mathrm{GeV}$ ), respectively. We tested the parameterized efficiency model in the CMSSM, and the results obtained agree at the $15 \%$ level with the full simulation results.

In summary, we conducted a search for physics beyond the standard model based on same-sign dileptons in the $e e$, $\mu \mu, e \mu, e \tau, \mu \tau$, and $\tau \tau$ final states, and find no evidence for an excess over the expected standard model background. We set $95 \%$ CL upper limits on contributions from new physics processes based on an integrated luminosity of $4.98 \mathrm{fb}^{-1}$ in the range of 6.2 to 16.9 events, depending on the signal search region. These are the most restrictive limits in this particular final state to date. We have also shown the excluded region in the CMSSM parameter space.

We wish to congratulate our colleagues in the CERN accelerator departments for the excellent performance of the LHC machine. We thank the technical and administrative staff at CERN and other CMS institutes, and acknowledge support from: FMSR (Austria); FNRS and FWO (Belgium); CNPq, CAPES, FAPERJ, and FAPESP (Brazil); MES (Bulgaria); CERN; CAS, MoST, and NSFC (China); COLCIENCIAS (Colombia); MSES (Croatia); RPF (Cyprus); Academy of Sciences and NICPB (Estonia); Academy of Finland, MEC, and HIP (Finland); CEA and CNRS/IN2P3 (France); BMBF, DFG, and HGF (Germany); GSRT (Greece); OTKA and NKTH (Hungary); DAE and DST (India); IPM (Iran); SFI (Ireland); INFN (Italy); NRF and WCU (Korea); LAS (Lithuania); CINVESTAV, CONACYT, SEP, and UASLP-FAI (Mexico); MSI (New Zealand); PAEC (Pakistan); SCSR (Poland); FCT (Portugal); JINR
(Armenia, Belarus, Georgia, Ukraine, Uzbekistan); MST, MAE and RFBR (Russia); MSTD (Serbia); MICINN and CPAN (Spain); Swiss Funding Agencies (Switzerland); NSC (Taipei); TUBITAK and TAEK (Turkey); STFC (United Kingdom); DOE and NSF (USA).

[1] P. Fayet and S. Ferrara, Phys. Rep. 32, 249 (1977).

[2] G. Aad et al. (ATLAS Collaboration), J. High Energy Phys. 11 (2011) 099.

[3] S. Chatrchyan et al. (CMS Collaboration), Phys. Rev. Lett. 107, 221804 (2011).

[4] G. Aad et al. (ATLAS Collaboration), Phys. Rev. D 85, 012006 (2012).

[5] S. Chatrchyan et al. (CMS Collaboration), J. High Energy Phys. 08 (2011) 156.

[6] G. Aad et al. (ATLAS Collaboration), Phys. Lett. B 709, 137 (2012).

[7] S. Chatrchyan et al. (CMS Collaboration), J. High Energy Phys. 06 (2011) 026.

[8] S. Chatrchyan et al. (CMS Collaboration), J. High Energy Phys. 06 (2011) 077.

[9] S. Chatrchyan et al. (CMS Collaboration), Phys. Lett. B 704, 411 (2011).

[10] G. Aad et al. (ATLAS Collaboration), Phys. Lett. B 710, 519 (2012).

[11] S. Chatrchyan et al. (CMS Collaboration), Phys. Rev. Lett. 106, 211802 (2011).

[12] R. M. Barnett, J. F. Gunion, and H. E. Haber, Phys. Lett. B 315, 349 (1993).

[13] M. Guchait and D. P. Roy, Phys. Rev. D 52, 133 (1995).

[14] H. Baer, C.-h. Chen, F. Paige, and X. Tata, Phys. Rev. D 53, 6241 (1996).

[15] H. C. Cheng, K. T. Matchev, and M. Schmaltz, Phys. Rev. D 66, 056006 (2002).

[16] S. Jung, H. Murayama, and A. Pierce, Phys. Rev. D 81, 015004 (2010).

[17] E. L. Berger, Q.-H. Cao, C.-R. Chen, S. L. Chong, and H. Zhang, Phys. Rev. Lett. 106, 201801 (2011).

[18] R. Contino and G. Servant, J. High Energy Phys. 06 (2008) 026.

[19] W.-Y. Keung and G. Senjanovic, Phys. Rev. Lett. 50, 1427 (1983).

[20] Y. Bai and Z. Han, J. High Energy Phys. 04 (2009) 056.

[21] S. Chatrchyan et al. (CMS Collaboration), JINST 3, S08004 (2008).

[22] G. L. Kane, C. F. Kolda, L. Roszkowski, and J. D. Wells, Phys. Rev. D 49, 6173 (1994).

[23] CMS Collaboration, CMS Physics Analysis Summary Report No. CMS-PAS-MUO-10-002, 2010.

[24] CMS Collaboration, CMS Physics Analysis Summary Report No. CMS-PAS-EGM-10-004, 2010.

[25] S. Chatrchyan (CMS), JINST 7, P01001 (2012).

[26] CMS Collaboration, CMS Physics Analysis Summary Report No. CMS-PAS-PFT- 09-001, 2009.

[27] CMS Collaboration, CMS Physics Analysis Summary Report No. CMS-PAS-PFT-10-002, 2010. 
[28] M. Cacciari, G. P. Salam, and G. Soyez, J. High Energy Phys. 04 2008) 063.

[29] J. Alwall, P. Demin, S. Visscher, R. Frederix, M. Herquet, F. Maltoni, T. Plehn, D. L. Rainwater, and T. Stelzer, J. High Energy Phys. 09 (2007) 028.

[30] T. Sjöstrand, S. Mrenna, and P. Skands, J. High Energy Phys. 05 (2006) 026.

[31] CMS Collaboration, J. Phys. G 34, 995 (2007).

[32] CMS Collaboration (CMS), CMS Physics Analysis Summary Report No. CMS-PAS-SMP-12-008, 2012.

[33] A. L. Read, J. Phys. G 28, 2693 (2002).

[34] T. Junk, Nucl. Instrum. Methods Phys. Res., Sect. A 434, 435 (1999).

[35] ATLAS and CMS Collaborations, LHC Higgs Combination Group, Report No. ATL-PHYS-PUB 201111, CMS NOTE 2011-005, 2011.

[36] M. Kramer, A. Kulesza, R. van der Leeuw, M. Mangano, and S. Padhi et al., arXiv:1206.2892.
[37] W. Beenakker, R. Höpker, M. Spira, and P. M. Zerwas, Nucl. Phys. B492, 51 (1997).

[38] A. Kulesza and L. Motyka, Phys. Rev. Lett. 102, 111802 (2009).

[39] A. Kulesza and L. Motyka, Phys. Rev. D 80, 095004 (2009).

[40] W. Beenakker, S. Brensing, M. Kramer, A. Kulesza, and E. Laenen et al., J. High Energy Phys. 12 (2009) 041.

[41] W. Beenakker, S. Brensing, M. Kramer, A. Kulesza, and E. Laenen et al., Int. J. Mod. Phys. A 26, 2637 (2011).

[42] W. Beenakker, M. Kramer, T. Plehn, M. Spira, and P. M. Zerwas, Nucl. Phys. B515, 3 (1998).

[43] W. Beenakker, S. Brensing, M. Kramer, A. Kulesza, and E. Laenen et al., J. High Energy Phys. 08 (2010) 098.

[44] W. Beenakker, M. Klasen, M. Kramer, T. Plehn, and M. Spira et al., Phys. Rev. Lett. 83, 3780 (1999).

S. Chatrchyan, ${ }^{1}$ V. Khachatryan, ${ }^{1}$ A. M. Sirunyan, ${ }^{1}$ A. Tumasyan, ${ }^{1}$ W. Adam, ${ }^{2}$ T. Bergauer, ${ }^{2}$ M. Dragicevic, ${ }^{2}$ J. Erö, ${ }^{2}$ C. Fabjan, ${ }^{2, b}$ M. Friedl, ${ }^{2}$ R. Frühwirth, ${ }^{2, b}$ V. M. Ghete, ${ }^{2}$ J. Hammer, ${ }^{2}$ N. Hörmann, ${ }^{2}$ J. Hrubec, ${ }^{2}$ M. Jeitler, ${ }^{2, b}$ W. Kiesenhofer, ${ }^{2}$ V. Knünz, ${ }^{2}$ M. Krammer, ${ }^{2, b}$ D. Liko, ${ }^{2}$ I. Mikulec, ${ }^{2}$ M. Pernicka, ${ }^{2, a}$ B. Rahbaran, ${ }^{2}$ C. Rohringer, ${ }^{2}$ H. Rohringer, ${ }^{2}$ R. Schöfbeck, ${ }^{2}$ J. Strauss, ${ }^{2}$ A. Taurok, ${ }^{2}$ P. Wagner, ${ }^{2}$ W. Waltenberger, ${ }^{2}$ G. Walzel, ${ }^{2}$ E. Widl, ${ }^{2}$ C.-E. Wulz, ${ }^{2, b}$ V. Mossolov, ${ }^{3}$ N. Shumeiko, ${ }^{3}$ J. Suarez Gonzalez, ${ }^{3}$ S. Bansal,${ }^{4}$ T. Cornelis,${ }^{4}$ E. A. De Wolf, ${ }^{4}$ X. Janssen, ${ }^{4}$ S. Luyckx,${ }^{4}$ T. Maes, ${ }_{4}^{4}$ L. Mucibello, ${ }^{4}$ S. Ochesanu, ${ }^{4}$ B. Roland,${ }^{4}$ R. Rougny,${ }^{4}$ M. Selvaggi, ${ }^{4}$ Z. Staykova, ${ }^{4}$ H. Van Haevermaet, ${ }^{4}$ P. Van Mechelen, ${ }^{4}$ N. Van Remortel, ${ }^{4}$ A. Van Spilbeeck, ${ }^{4}$ F. Blekman, ${ }^{5}$ S. Blyweert, ${ }^{5}$ J. D'Hondt, ${ }^{5}$ R. Gonzalez Suarez, ${ }^{5}$ A. Kalogeropoulos, ${ }^{5}$ M. Maes, ${ }^{5}$ A. Olbrechts, ${ }^{5}$ W. Van Doninck, ${ }^{5}$ P. Van Mulders, ${ }^{5}$ G. P. Van Onsem, ${ }^{5}$ I. Villella, ${ }^{5}$ O. Charaf, ${ }^{6}$ B. Clerbaux,${ }^{6}$ G. De Lentdecker, ${ }^{6}$ V. Dero, ${ }^{6}$ A. P. R. Gay, ${ }^{6}$ T. Hreus, ${ }^{6}$ A. Léonard, ${ }^{6}$ P. E. Marage, ${ }^{6}$ T. Reis, ${ }^{6}$ L. Thomas, ${ }^{6}$ C. Vander Velde,${ }^{6}$ P. Vanlaer, ${ }^{6}$ J. Wang, ${ }^{6}$ V. Adler, ${ }^{7}$

K. Beernaert, ${ }^{7}$ A. Cimmino, ${ }^{7}$ S. Costantini, ${ }^{7}$ G. Garcia, ${ }^{7}$ M. Grunewald ${ }^{7}$ B. Klein, ${ }^{7}$ J. Lellouch, ${ }^{7}$ A. Marinov, ${ }^{7}$

J. Mccartin, ${ }^{7}$ A. A. Ocampo Rios, ${ }^{7}$ D. Ryckbosch,${ }^{7}$ N. Strobbe,${ }^{7}$ F. Thyssen, ${ }^{7}$ M. Tytgat, ${ }^{7}$ L. Vanelderen, ${ }^{7}$

P. Verwilligen, ${ }^{7}$ S. Walsh, ${ }^{7}$ E. Yazgan, ${ }^{7}$ N. Zaganidis, ${ }^{7}$ S. Basegmez,${ }^{8}$ G. Bruno, ${ }^{8}$ R. Castello, ${ }^{8}$ A. Caudron, ${ }^{8}$ L. Ceard, ${ }^{8}$ C. Delaere, ${ }^{8}$ T. du Pree, ${ }^{8}$ D. Favart, ${ }^{8}$ L. Forthomme, ${ }^{8}$ A. Giammanco,${ }^{8, \mathrm{c}}$ J. Hollar, ${ }^{8}$ V. Lemaitre, ${ }^{8}$ J. Liao, ${ }^{8}$ O. Militaru, ${ }^{8}$ C. Nuttens, ${ }^{8}$ D. Pagano, ${ }^{8}$ L. Perrini, ${ }^{8}$ A. Pin,${ }^{8}$ K. Piotrzkowski, ${ }^{8}$ N. Schul, ${ }^{8}$ J. M. Vizan Garcia, ${ }^{8}$ N. Beliy, ${ }^{9}$ T. Caebergs, ${ }^{9}$ E. Daubie, ${ }^{9}$ G. H. Hammad, ${ }^{9}$ G. A. Alves,${ }^{10}$ M. Correa Martins Junior, ${ }^{10}$

D. De Jesus Damiao, ${ }^{10}$ T. Martins,${ }^{10}$ M.E. Pol, ${ }^{10}$ M. H. G. Souza,${ }^{10}$ W. L. Aldá Júnior ${ }^{11}$ W. Carvalho,${ }^{11}$

A. Custódio, ${ }^{11}$ E. M. Da Costa, ${ }^{11}$ C. De Oliveira Martins, ${ }^{11}$ S. Fonseca De Souza,${ }^{11}$ D. Matos Figueiredo,,${ }^{11}$ L. Mundim, ${ }^{11}$ H. Nogima, ${ }^{11}$ V. Oguri, ${ }^{11}$ W. L. Prado Da Silva, ${ }^{11}$ A. Santoro, ${ }^{11}$ L. Soares Jorge, ${ }^{11}$ A. Sznajder, ${ }^{11}$ C. A. Bernardes, ${ }^{12, d}$ F. A. Dias, ${ }^{12, e}$ T. R. Fernandez Perez Tomei, ${ }^{12}$ E. M. Gregores, ${ }^{12, d}$ C. Lagana, ${ }^{12}$ F. Marinho, ${ }^{12}$ P. G. Mercadante, ${ }^{12, \mathrm{~d}}$ S. F. Novaes ${ }^{12}$ Sandra S. Padula, ${ }^{12}$ V. Genchev, ${ }^{13, f}$ P. Iaydjiev, ${ }^{13, f}$ S. Piperov, ${ }^{13}$ M. Rodozov, ${ }^{13}$

S. Stoykova, ${ }^{13}$ G. Sultanov, ${ }^{13}$ V. Tcholakov, ${ }^{13}$ R. Trayanov, ${ }^{13}$ M. Vutova, ${ }^{13}$ A. Dimitrov,${ }^{14}$ R. Hadjiiska, ${ }^{14}$ V. Kozhuharov, ${ }^{14}$ L. Litov, ${ }^{14}$ B. Pavlov, ${ }^{14}$ P. Petkov, ${ }^{14}$ J. G. Bian, ${ }^{15}$ G. M. Chen,${ }^{15}$ H. S. Chen, ${ }^{15}$ C. H. Jiang, ${ }^{15}$ D. Liang, ${ }^{15}$ S. Liang, ${ }^{15}$ X. Meng, ${ }^{15}$ J. Tao, ${ }^{15}$ J. Wang, ${ }^{15}$ X. Wang,${ }^{15}$ Z. Wang,${ }^{15}$ H. Xiao, ${ }^{15}$ M. Xu, ${ }^{15}$ J. Zang, ${ }^{15}$ Z. Zhang, ${ }^{15}$ C. Asawatangtrakuldee, ${ }^{16}$ Y. Ban,${ }^{16}$ S. Guo, ${ }^{16}$ Y. Guo, ${ }^{16} \mathrm{~W}$. Li, ${ }^{16}$ S. Liu, ${ }^{16}$ Y. Mao, ${ }^{16}$ S. J. Qian, ${ }^{16}$ H. Teng, ${ }^{16}$ S. Wang, ${ }^{16}$ B. Zhu, ${ }^{16}$ W. Zou, ${ }^{16}$ C. Avila, ${ }^{17}$ J. P. Gomez, ${ }^{17}$ B. Gomez Moreno, ${ }^{17}$ A. F. Osorio Oliveros, ${ }^{17}$ J. C. Sanabria, ${ }^{17}$ N. Godinovic, ${ }^{18}$ D. Lelas, ${ }^{18}$ R. Plestina, ${ }^{18, \mathrm{~g}}$ D. Polic, ${ }^{18}$ I. Puljak, ${ }^{18, f}$ Z. Antunovic, ${ }^{19}$ M. Kovac, ${ }^{19}$ V. Brigljevic, ${ }^{20}$ S. Duric,${ }^{20}$ K. Kadija, ${ }^{20}$ J. Luetic, ${ }^{20}$ S. Morovic, ${ }^{20}$ A. Attikis, ${ }^{21}$ M. Galanti, ${ }^{21}$ G. Mavromanolakis,${ }^{21}$ J. Mousa, ${ }^{21}$ C. Nicolaou, ${ }^{21}$ F. Ptochos, ${ }^{21}$ P. A. Razis,${ }^{21}$ M. Finger,${ }^{22}$ M. Finger, Jr., ${ }^{22}$ Y. Assran, ${ }^{23, h}$ S. Elgammal,${ }^{23, i}$ A. Ellithi Kamel, ${ }^{23, j}$ S. Khalil, ${ }^{23, i}$ M. A. Mahmoud, ${ }^{23, \mathrm{k}}$ A. Radi, ${ }^{23,1, e e e}$ M. Kadastik, ${ }^{24}$ M. Müntel, ${ }^{24}$ M. Raidal, ${ }^{24}$ L. Rebane, ${ }^{24}$ A. Tiko, ${ }^{24}$ V. Azzolini, ${ }^{25}$ P. Eerola,${ }^{25}$ G. Fedi, ${ }^{25}$ M. Voutilainen,${ }^{25}$ J. Härkönen, ${ }^{26}$ A. Heikkinen ${ }^{26}$ V. Karimäki, ${ }^{26}$ R. Kinnunen, ${ }^{26}$ M. J. Kortelainen, ${ }^{26}$ T. Lampén, ${ }^{26}$ K. Lassila-Perini,${ }^{26}$ S. Lehti, ${ }^{26}$ T. Lindén, ${ }^{26}$ P. Luukka, ${ }^{26}$ T. Mäenpää, ${ }^{26}$ T. Peltola, ${ }^{26}$ E. Tuominen, ${ }^{26}$ J. Tuominiemi ${ }^{26}$ E. Tuovinen, ${ }^{26}$ D. Ungaro,${ }^{26}$ L. Wendland, ${ }^{26}$ K. Banzuzi, ${ }^{27}$ A. Korpela,${ }^{27}$ T. Tuuva, ${ }^{27}$ M. Besancon, ${ }^{28}$ S. Choudhury, ${ }^{28}$ M. Dejardin, ${ }^{28}$ 
D. Denegri, ${ }^{28}$ B. Fabbro, ${ }^{28}$ J. L. Faure, ${ }^{28}$ F. Ferri, ${ }^{28}$ S. Ganjour ${ }^{28}$ A. Givernaud,${ }^{28}$ P. Gras, ${ }^{28}$

G. Hamel de Monchenault, ${ }^{28}$ P. Jarry, ${ }^{28}$ E. Locci, ${ }^{28}$ J. Malcles, ${ }^{28}$ L. Millischer ${ }^{28}$ A. Nayak, ${ }^{28}$ J. Rander,${ }^{28}$ A. Rosowsky, ${ }^{28}$ I. Shreyber, ${ }^{28}$ M. Titov, ${ }^{28}$ S. Baffioni, ${ }^{29}$ F. Beaudette, ${ }^{29}$ L. Benhabib, ${ }^{29}$ L. Bianchini, ${ }^{29}$ M. Bluj,${ }^{29, m}$ C. Broutin, ${ }^{29}$ P. Busson, ${ }^{29}$ C. Charlot, ${ }^{29}$ N. Daci, ${ }^{29}$ T. Dahms, ${ }^{29}$ L. Dobrzynski, ${ }^{29}$ R. Granier de Cassagnac, ${ }^{29}$ M. Haguenauer, ${ }^{29}$ P. Miné, ${ }^{29}$ C. Mironov, ${ }^{29}$ M. Nguyen,${ }^{29}$ C. Ochando,${ }^{29}$ P. Paganini, ${ }^{29}$ D. Sabes,${ }^{29}$ R. Salerno, ${ }^{29}$ Y. Sirois,${ }^{29}$ C. Veelken, ${ }^{29}$ A. Zabi, ${ }^{29}$ J.-L. Agram, ${ }^{30, n}$ J. Andrea, ${ }^{30}$ D. Bloch,${ }^{30}$ D. Bodin,${ }^{30}$ J.-M. Brom, ${ }^{30}$ M. Cardaci, ${ }^{30}$ E. C. Chabert, ${ }^{30}$ C. Collard, ${ }^{30}$ E. Conte,${ }^{30, n}$ F. Drouhin,,${ }^{30, n}$ C. Ferro,${ }^{30}$ J.-C. Fontaine, ${ }^{30, n}$ D. Gelé, ${ }^{30}$ U. Goerlach, ${ }^{30}$ P. Juillot,${ }^{30}$ M. Karim, ${ }^{30, n}$ A.-C. Le Bihan, ${ }^{30}$ P. Van Hove, ${ }^{30}$ F. Fassi, ${ }^{31}$ D. Mercier, ${ }^{31}$ S. Beauceron, ${ }^{32}$ N. Beaupere, ${ }^{32}$ O. Bondu, ${ }^{32}$ G. Boudoul,${ }^{32}$ H. Brun, ${ }^{32}$ J. Chasserat,${ }^{32}$ R. Chierici, ${ }^{32, f}$ D. Contardo, ${ }^{32}$ P. Depasse, ${ }^{32}$ H. El Mamouni, ${ }^{32}$ J. Fay, ${ }^{32}$ S. Gascon, ${ }^{32}$ M. Gouzevitch, ${ }^{32}$ B. Ille, ${ }^{32}$ T. Kurca, ${ }^{32}$ M. Lethuillier, ${ }^{32}$ L. Mirabito, ${ }^{32}$ S. Perries, ${ }^{32}$ V. Sordini, ${ }^{32}$ S. Tosi, ${ }^{32}$ Y. Tschudi, ${ }^{32}$ P. Verdier, ${ }^{32}$ S. Viret, ${ }^{32}$ Z. Tsamalaidze, ${ }^{33, y}$ G. Anagnostou, ${ }^{34}$

S. Beranek ${ }^{34}$ M. Edelhoff, ${ }^{34}$ L. Feld,${ }^{34}$ N. Heracleous, ${ }^{34}$ O. Hindrichs, ${ }^{34}$ R. Jussen, ${ }^{34}$ K. Klein, ${ }^{34}$ J. Merz, ${ }^{34}$ A. Ostapchuk, ${ }^{34}$ A. Perieanu, ${ }^{34}$ F. Raupach, ${ }^{34}$ J. Sammet, ${ }^{34}$ S. Schael, ${ }^{34}$ D. Sprenger, ${ }^{34}$ H. Weber, ${ }^{34}$ B. Wittmer, ${ }^{34}$ V. Zhukov, ${ }^{34,0}$ M. Ata, ${ }^{35}$ J. Caudron, ${ }^{35}$ E. Dietz-Laursonn, ${ }^{35}$ D. Duchardt, ${ }^{35}$ M. Erdmann, ${ }^{35}$ R. Fischer, ${ }^{35}$ A. Güth, ${ }^{35}$

T. Hebbeker, ${ }^{35}$ C. Heidemann, ${ }^{35}$ K. Hoepfner, ${ }^{35}$ D. Klingebiel, ${ }^{35}$ P. Kreuzer, ${ }^{35}$ J. Lingemann, ${ }^{35}$ C. Magass, ${ }^{35}$

M. Merschmeyer, ${ }^{35}$ A. Meyer, ${ }^{35}$ M. Olschewski, ${ }^{35}$ P. Papacz, ${ }^{35}$ H. Pieta, ${ }^{35}$ H. Reithler, ${ }^{35}$ S. A. Schmitz, ${ }^{35}$

L. Sonnenschein, ${ }^{35}$ J. Steggemann, ${ }^{35}$ D. Teyssier, ${ }^{35} \mathrm{M}$. Weber, ${ }^{35} \mathrm{M}$. Bontenackels,${ }^{36} \mathrm{~V}$. Cherepanov, ${ }^{36} \mathrm{M}$. Davids ${ }^{36}$ G. Flügge, ${ }^{36}$ H. Geenen, ${ }^{36}$ M. Geisler, ${ }^{36}$ W. Haj Ahmad, ${ }^{36}$ F. Hoehle, ${ }^{36}$ B. Kargoll, ${ }^{36}$ T. Kress, ${ }^{36}$ Y. Kuessel, ${ }^{36}$ A. Linn, ${ }^{36}$ A. Nowack, ${ }^{36}$ L. Perchalla ${ }^{36}$ O. Pooth,${ }^{36}$ J. Rennefeld, ${ }^{36}$ P. Sauerland, ${ }^{36}$ A. Stahl, ${ }^{36}$ M. Aldaya Martin, ${ }^{37}$ J. Behr,${ }^{37}$ W. Behrenhoff, ${ }^{37}$ U. Behrens, ${ }^{37}$ M. Bergholz, ${ }^{37, p}$ A. Bethani, ${ }^{37}$ K. Borras, ${ }^{37}$ A. Burgmeier,${ }^{37}$ A. Cakir, ${ }^{37}$ L. Calligaris, ${ }^{37}$ A. Campbell, ${ }^{37}$ E. Castro, ${ }^{37}$ F. Costanza, ${ }^{37}$ D. Dammann, ${ }^{37}$ G. Eckerlin, ${ }^{37}$ D. Eckstein, ${ }^{37}$ D. Fischer, ${ }^{37}$ G. Flucke, ${ }^{37}$ A. Geiser,${ }^{37}$ I. Glushkov, ${ }^{37}$ P. Gunnellini, ${ }^{37}$ S. Habib,${ }^{37}$ J. Hauk, ${ }^{37}$ G. Hellwig,,${ }^{37}$ H. Jung, ${ }^{37, f}$ M. Kasemann, ${ }^{37}$ P. Katsas, ${ }^{37}$ C. Kleinwort,${ }^{37}$ H. Kluge, ${ }^{37}$ A. Knutsson, ${ }^{37}$ M. Krämer, ${ }^{37}$ D. Krücker,,${ }^{37}$ E. Kuznetsova, ${ }^{37}$ W. Lange, ${ }^{37}$ W. Lohmann, ${ }^{37, p}$ B. Lutz,${ }^{37}$ R. Mankel,${ }^{37}$ I. Marfin, ${ }^{37}$ M. Marienfeld, ${ }^{37}$ I.-A. Melzer-Pellmann, ${ }^{37}$ A. B. Meyer ${ }^{37}$ J. Mnich, ${ }^{37}$ A. Mussgiller,${ }^{37}$ S. Naumann-Emme, ${ }^{37}$ J. Olzem,${ }^{37}$ H. Perrey, ${ }^{37}$ A. Petrukhin, ${ }^{37}$ D. Pitzl, ${ }^{37}$ A. Raspereza, ${ }^{37}$ P. M. Ribeiro Cipriano, ${ }^{37}$ C. Riedl,${ }^{37}$ M. Rosin, ${ }^{37}$ J. Salfeld-Nebgen, ${ }^{37}$ R. Schmidt, ${ }^{37, p}$ T. Schoerner-Sadenius, ${ }^{37}$ N. Sen, ${ }^{37}$ A. Spiridonov, ${ }^{37}$ M. Stein, ${ }^{37}$ R. Walsh,${ }^{37}$ C. Wissing,,${ }^{37}$ C. Autermann, ${ }^{38}$ V. Blobel, ${ }^{38}$ S. Bobrovskyi, ${ }^{38}$ J. Draeger, ${ }^{38}$ H. Enderle, ${ }^{38}$ J. Erfle,${ }^{38}$ U. Gebbert, ${ }^{38}$ M. Görner, ${ }^{38}$ T. Hermanns, ${ }^{38}$ R. S. Höing, ${ }^{38}$ K. Kaschube, ${ }^{38}$ G. Kaussen,${ }^{38}$ H. Kirschenmann, ${ }^{38}$ R. Klanner, ${ }^{38}$ J. Lange,${ }^{38}$ B. Mura, ${ }^{38}$ F. Nowak, ${ }^{38}$ T. Peiffer, ${ }^{38}$ N. Pietsch, ${ }^{38}$ D. Rathjens, ${ }^{38}$ C. Sander, ${ }^{38}$ H. Schettler, ${ }^{38}$ P. Schleper, ${ }^{38}$ E. Schlieckau, ${ }^{38}$ A. Schmidt, ${ }^{38}$ M. Schröder,${ }^{38}$ T. Schum, ${ }^{38}$ M. Seidel,${ }^{38}$ H. Stadie,${ }^{38}$ G. Steinbrück,${ }^{38}$ J. Thomsen, ${ }^{38}$ C. Barth ${ }^{39}$ J. Berger, ${ }^{39}$ C. Böser, ${ }^{39}$ T. Chwalek, ${ }^{39}$ W. De Boer,${ }^{39}$ A. Descroix,${ }^{39}$ A. Dierlamm, ${ }^{39}$ M. Feindt, ${ }^{39}$ M. Guthoff, ${ }^{39, f}$ C. Hackstein, ${ }^{39}$ F. Hartmann, ${ }^{39}$ T. Hauth,,${ }^{39, f}$ M. Heinrich, ${ }^{39}$ H. Held, ${ }^{39}$ K. H. Hoffmann, ${ }^{39}$ S. Honc, ${ }^{39}$ I. Katkov, ${ }^{39,0}$ J. R. Komaragiri, ${ }^{39}$ D. Martschei, ${ }^{39}$ S. Mueller ${ }^{39}$ Th. Müller, ${ }^{39}$ M. Niegel, ${ }^{39}$ A. Nürnberg, ${ }^{39}$ O. Oberst, ${ }^{39}$ A. Oehler, ${ }^{39}$ J. Ott, ${ }^{39}$ G. Quast,${ }^{39}$ K. Rabbertz,${ }^{39}$ F. Ratnikov, ${ }^{39}$ N. Ratnikova, ${ }^{39}$ S. Röcker, ${ }^{39}$ A. Scheurer, ${ }^{39}$ F.-P. Schilling, ${ }^{39}$ G. Schott, ${ }^{39}$ H. J. Simonis, ${ }^{39}$ F. M. Stober, ${ }^{39}$ D. Troendle, ${ }^{39}$ R. Ulrich, ${ }^{39}$ J. Wagner-Kuhr, ${ }^{39}$ S. Wayand, ${ }^{39}$ T. Weiler, ${ }^{39}$ M. Zeise, ${ }^{39}$ G. Daskalakis, ${ }^{40}$ T. Geralis, ${ }^{40}$ S. Kesisoglou, ${ }^{40}$ A. Kyriakis, ${ }^{40}$ D. Loukas, ${ }^{40}$ I. Manolakos, ${ }^{40}$ A. Markou, ${ }^{40}$ C. Markou, ${ }^{40}$ C. Mavrommatis, ${ }^{40}$ E. Ntomari, ${ }^{40}$ L. Gouskos ${ }^{41}$ T. J. Mertzimekis ${ }^{41}$ A. Panagiotou, ${ }^{41}$ N. Saoulidou, ${ }^{41}$ I. Evangelou, ${ }^{42}$ C. Foudas,${ }^{42, f}$ P. Kokkas, ${ }^{42}$ N. Manthos ${ }^{42}$ I. Papadopoulos, ${ }^{42}$ V. Patras, ${ }^{42}$ G. Bencze, ${ }^{43}$ C. Hajdu, ${ }^{43, f}$ P. Hidas, ${ }^{43}$ D. Horvath,${ }^{43, q}$ K. Krajczar, ${ }^{43, r}$ B. Radics, ${ }^{43}$ F. Sikler, ${ }^{43, \mathrm{f}}$ V. Veszpremi, ${ }^{43}$ G. Vesztergombi, ${ }^{43, \mathrm{r}}$ N. Beni, ${ }^{44}$ S. Czellar, ${ }^{44}$ J. Molnar, ${ }^{44}$ J. Palinkas,${ }^{44}$ Z. Szillasi, ${ }^{44}$ J. Karancsi, ${ }^{45}$ P. Raics, ${ }^{45}$ Z. L. Trocsanyi, ${ }^{45}$ B. Ujvari, ${ }^{45}$ S. B. Beri, ${ }^{46}$ V. Bhatnagar, ${ }^{46}$ N. Dhingra, ${ }^{46}$ R. Gupta, ${ }^{46}$ M. Jindal, ${ }^{46}$ M. Kaur, ${ }^{46}$ J. M. Kohli, ${ }^{46}$ M. Z. Mehta, ${ }^{46}$ N. Nishu, ${ }^{46}$ L. K. Saini, ${ }^{46}$ A. Sharma, ${ }^{46}$ J. Singh ${ }^{46}$ S. Ahuja, ${ }^{47}$ A. Bhardwaj,${ }^{47}$ B. C. Choudhary, ${ }^{47}$ A. Kumar,${ }^{47}$ A. Kumar,${ }^{47}$ S. Malhotra, ${ }^{47}$ M. Naimuddin, ${ }^{47}$ K. Ranjan, ${ }^{47}$ V. Sharma ${ }^{47}$ R. K. Shivpuri, ${ }^{47}$ S. Banerjee, ${ }^{48}$ S. Bhattacharya ${ }^{48}$ S. Dutta, ${ }^{48}$ B. Gomber ${ }^{48}$ Sa. Jain, ${ }^{48}$ Sh. Jain,${ }^{48}$ R. Khurana, ${ }^{48}$ S. Sarkar, ${ }^{48}$ M. Sharan, ${ }^{48}$ A. Abdulsalam, ${ }^{49}$ R. K. Choudhury, ${ }^{49}$ D. Dutta, ${ }^{49}$ S. Kailas,${ }^{49}$ V. Kumar, ${ }^{49}$ P. Mehta, ${ }^{49}$ A. K. Mohanty, ${ }^{49, f}$ L. M. Pant, ${ }^{49}$ P. Shukla, ${ }^{49}$ T. Aziz,${ }^{50}$ S. Ganguly, ${ }^{50}$ M. Guchait, ${ }^{50, s}$ M. Maity ${ }^{50, t}$ G. Majumder, ${ }^{50}$ K. Mazumdar,${ }^{50}$ G. B. Mohanty,${ }^{50}$ B. Parida,${ }^{50}$ K. Sudhakar, ${ }^{50}$ N. Wickramage,${ }^{50}$ S. Banerjee, ${ }^{51}$ S. Dugad,${ }^{51}$ H. Arfaei,${ }^{52}$ H. Bakhshiansohi, ${ }^{52, \mathrm{u}}$ S. M. Etesami, ${ }^{52, \mathrm{v}}$ A. Fahim, ${ }^{52, \mathrm{u}}$ M. Hashemi, ${ }^{52}$ H. Hesari, ${ }^{52}$ A. Jafari, ${ }^{52, \mathrm{u}}$ M. Khakzad,${ }^{52}$ A. Mohammadi, ${ }^{52, \mathrm{w}}$ M. Mohammadi Najafabadi, ${ }^{52}$ S. Paktinat Mehdiabadi, ${ }^{52}$ B. Safarzadeh,${ }^{52, \mathrm{x}}$ M. Zeinali, ${ }^{52, \mathrm{v}}$ M. Abbrescia, ${ }^{53 a, 53 b}$ L. Barbone, ${ }^{53 a, 53 b}$ 
C. Calabria, ${ }^{53 a, 53 b, f}$ S. S. Chhibra, ${ }^{53 a, 53 b}$ A. Colaleo, ${ }^{53 a}$ D. Creanza, ${ }^{53 a, 53 c}$ N. De Filippis, ${ }^{53 a, 53 c, f}$ M. De Palma, ${ }^{53 a, 53 b}$ L. Fiore,${ }^{53 a}$ G. Iaselli, ${ }^{53 a, 53 c}$ L. Lusito, ${ }^{53 a, 53 b}$ G. Maggi, ${ }^{53 a, 53 c}$ M. Maggi, ${ }^{53 a}$ B. Marangelli, ${ }^{53 a, 53 b}$ S. My, ${ }^{53 a, 53 c}$ S. Nuzzo, ${ }^{53 a, 53 b}$ N. Pacifico, ${ }^{53 a, 53 b}$ A. Pompili, ${ }^{53 a, 53 b}$ G. Pugliese, ${ }^{53 a, 53 c}$ G. Selvaggi, ${ }^{53 a, 53 b}$ L. Silvestris, ${ }^{53 a}$ G. Singh, ${ }^{53 a, 53 b}$ R. Venditti, ${ }^{53 a}$ G. Zito, ${ }^{53 a}$ G. Abbiendi, ${ }^{54 a}$ A. C. Benvenuti, ${ }^{54 a}$ D. Bonacorsi, ${ }^{54 a, 54 b}$

S. Braibant-Giacomelli, ${ }^{54 a, 54 b}$ L. Brigliadori, ${ }^{54,54 b}$ P. Capiluppi, ${ }^{54 a, 54 b}$ A. Castro, ${ }^{54 a, 54 b}$ F. R. Cavallo, ${ }^{54 a}$ M. Cuffiani, ${ }^{54,54 b}$ G. M. Dallavalle, ${ }^{54 a}$ F. Fabbri, ${ }^{54 a}$ A. Fanfani, ${ }^{54 a, 54 b}$ D. Fasanella, ${ }^{54 a, 54 b, f}$ P. Giacomelli, ${ }^{54 a}$ C. Grandi, ${ }^{54 a}$ L. Guiducci, ${ }^{54 a}$ S. Marcellini, ${ }^{54 a}$ G. Masetti ${ }^{54 a}$ M. Meneghelli, ${ }^{54 a, 54 b, f}$ A. Montanari, ${ }^{54 a}$ F. L. Navarria, ${ }^{54 a, 54 b}$ F. Odorici, ${ }^{54 a}$ A. Perrotta, ${ }^{54 a}$ F. Primavera, ${ }^{54 a, 54 b}$ A. M. Rossi, ${ }^{54 a, 54 b}$ T. Rovelli, ${ }^{54 a, 54 b}$ G. Siroli, ${ }^{54 a, 54 b}$ R. Travaglini, ${ }^{54 a, 54 b}$ S. Albergo, ${ }^{55 a, 55 b}$ G. Cappello, ${ }^{55 a, 55 b}$ M. Chiorboli, ${ }^{55 a, 55 b}$ S. Costa, ${ }^{55 a, 55 b}$ R. Potenza, ${ }^{55 a, 55 b}$ A. Tricomi, ${ }^{55 a, 55 b}$ C. Tuve,${ }^{55 a, 55 b}$ G. Barbagli, ${ }^{56 a}$ V. Ciulli, ${ }^{56 a, 56 b}$ C. Civinini, ${ }^{56 a}$ R. D'Alessandro, ${ }^{56,56 b}$ E. Focardi ${ }^{56 a, 56 b}$ S. Frosali, ${ }^{56 a, 56 b}$ E. Gallo, ${ }^{56 a}$ S. Gonzi, ${ }^{56 a, 56 b}$ M. Meschini ${ }^{56 a}$ S. Paoletti, ${ }^{56 a}$ G. Sguazzoni, ${ }^{56 a}$ A. Tropiano, ${ }^{56 a, f}$ L. Benussi, ${ }^{57}$ S. Bianco,${ }^{57}$ S. Colafranceschi, ${ }^{57, y}$ F. Fabbri, ${ }^{57}$ D. Piccolo, ${ }^{57}$ P. Fabbricatore,${ }^{58}$ R. Musenich, ${ }^{58}$ A. Benaglia, ${ }^{59 a, 59 b, f}$ F. De Guio,${ }^{59 a, 59 b}$ L. Di Matteo,,${ }^{59 a, 59 b, f}$ S. Fiorendi, ${ }^{59 a, 59 b}$ S. Gennai, ${ }^{59 a, f}$ A. Ghezzi, ${ }^{59 a, 59 b}$ S. Malvezzi,${ }^{59 a}$ R. A. Manzoni,${ }^{59 a, 59 b}$ A. Martelli, ${ }^{59 a, 59 b}$ A. Massironi, ${ }^{59 a, 59 b, f}$ D. Menasce, ${ }^{59 a}$ L. Moroni, ${ }^{59 a}$ M. Paganoni, ${ }^{59 a, 59 b}$ D. Pedrini, ${ }^{59 a}$ S. Ragazzi, ${ }^{59 a, 59 b}$ N. Redaelli, ${ }^{59 a}$ S. Sala, ${ }^{59 a}$

T. Tabarelli de Fatis, ${ }^{59 a, 59 b}$ S. Buontempo, ${ }^{60 a}$ C. A. Carrillo Montoya ${ }^{60 a, f}$ N. Cavallo, ${ }^{60 a, z}$ A. De Cosa,${ }^{60,60 b, f}$ O. Dogangun, ${ }^{60 a, 60 b}$ F. Fabozzi, ${ }^{60 a, z}$ A. O. M. Iorio, ${ }^{60 a}$ L. Lista ${ }^{60 a}$ S. Meola, ${ }^{60 a, a a}$ M. Merola, ${ }^{60 a, 60 b}$ P. Paolucci, ${ }^{60 a, f}$ P. Azzi ${ }^{61 \mathrm{a}}$ N. Bacchetta, ${ }^{61 \mathrm{a}, \mathrm{f}}$ P. Bellan, ${ }^{61 \mathrm{a}, 61 \mathrm{~b}}$ A. Branca,${ }^{61 \mathrm{a}, \mathrm{f}}$ R. Carlin, ${ }^{61 \mathrm{a}, 61 \mathrm{~b}}$ P. Checchia, ${ }^{61 \mathrm{a}} \mathrm{T}$. Dorigo, ${ }^{61 \mathrm{a}}$ U. Dosselli, ${ }^{61 \mathrm{a}}$ F. Gasparini, ${ }^{61 \mathrm{a}, 61 \mathrm{~b}}$ U. Gasparini, ${ }^{61 \mathrm{a}, 61 \mathrm{~b}}$ A. Gozzelino, ${ }^{61 \mathrm{a}} \mathrm{K}$. Kanishchev, ${ }^{61 \mathrm{a}, 61 \mathrm{c}} \mathrm{S}$. Lacaprara, ${ }^{61 \mathrm{a}}$ I. Lazzizzera, ${ }^{61 \mathrm{a}, 61 \mathrm{c}}$ M. Margoni, ${ }^{61 \mathrm{a}, 61 \mathrm{~b}}$ A. T. Meneguzzo, ${ }^{61 \mathrm{a}, 61 \mathrm{~b}}$ J. Pazzini, ${ }^{61 \mathrm{a}}$ L. Perrozzi, ${ }^{61 \mathrm{a}}$ N. Pozzobon, ${ }^{61 \mathrm{a}, 61 \mathrm{~b}}$ P. Ronchese ${ }^{61 \mathrm{a}, 61 \mathrm{~b}}$ F. Simonetto ${ }^{61 \mathrm{a}, 61 \mathrm{~b}}$ E. Torassa, ${ }^{61 \mathrm{a}}$ M. Tosi, ${ }^{61 \mathrm{a}, 61 \mathrm{~b}, \mathrm{f}}$ S. Vanini, ${ }^{61 \mathrm{a}, 61 \mathrm{~b}}$ A. Zucchetta, ${ }^{61 \mathrm{a}}$ G. Zumerle, ${ }^{61 a, 61 b}$ M. Gabusi, ${ }^{62 a, 62 b}$ S. P. Ratti, ${ }^{62 a, 62 b}$ C. Riccardi, ${ }^{62 a, 62 b}$ P. Torre, ${ }^{62 a, 62 b}$ P. Vitulo, ${ }^{62 a, 62 b}$ M. Biasini, ${ }^{63 a, 63 b}$ G. M. Bilei, ${ }^{63 a}$ L. Fanò, ${ }^{63 a, 63 b}$ P. Lariccia, ${ }^{63 a, 63 b}$ A. Lucaroni, ${ }^{63 a, 63 b, f}$ G. Mantovani, ${ }^{63 a, 63 b}$ M. Menichelli, ${ }^{63 a}$ A. Nappi ${ }^{63 a, 63 b}$ F. Romeo, ${ }^{63 a, 63 b}$ A. Saha, ${ }^{63 a}$ A. Santocchia, ${ }^{63 a, 63 b}$ S. Taroni, ${ }^{63 a, 63 b, f}$ P. Azzurri, ${ }^{64 a, 64 c}$ G. Bagliesi, ${ }^{64 a}$ T. Boccali, ${ }^{64 a}$ G. Broccolo, ${ }^{64 a, 64 c}$ R. Castaldi, ${ }^{64 a}$ R. T. D'Agnolo, ${ }^{64 a, 64 c}$ R. Dell'Orso, ${ }^{64 a}$ F. Fiori, ${ }^{64 a, 64 b, f}$ L. Foà, ${ }^{64 a, 64 c}$ A. Giassi, ${ }^{64 a}$ A. Kraan,${ }^{64 a}$ F. Ligabue, ${ }^{64 a, 64 c}$ T. Lomtadze,${ }^{64 a}$ L. Martini, ${ }^{64 a, b b}$ A. Messineo, ${ }^{64 a, 64 b}$ F. Palla ${ }^{64 a}$ A. Rizzi, ${ }^{64 a, 64 b}$ A. T. Serban,${ }^{64 a, c c}$ P. Spagnolo, ${ }^{64 a}$ P. Squillacioti, ${ }^{64 a, f}$ R. Tenchini, ${ }^{64 a}$ G. Tonelli, ${ }^{64 a, 64 b, f}$ A. Venturi, ${ }^{64 a, f}$ P. G. Verdini, ${ }^{64 a}$ L. Barone,${ }^{65 a, 65 b}$ F. Cavallari, ${ }^{65 a}$ D. Del Re,${ }^{65 a, 65 b, f}$ M. Diemoz, ${ }^{65 a}$ M. Grassi, ${ }^{65 a, 65 b, f}$ E. Longo, ${ }^{65 a, 65 b}$ P. Meridiani, ${ }^{65 a, f}$ F. Micheli, ${ }^{65 a, 65 b}$ S. Nourbakhsh, ${ }^{65 a, 65 b}$ G. Organtini, ${ }^{65 a, 65 b}$ R. Paramatti, ${ }^{65 a}$ S. Rahatlou, ${ }^{65 a, 65 b}$ M. Sigamani, ${ }^{65 a}$ L. Soffi, ${ }^{65 a, 65 b}$ N. Amapane, ${ }^{66 a, 66 b}$ R. Arcidiacono ${ }^{66 \mathrm{a}, 66 \mathrm{c}}$ S. Argiro, ${ }^{66 \mathrm{a}, 66 \mathrm{~b}} \mathrm{M}$. Arneodo, ${ }^{66 \mathrm{a}, 66 \mathrm{c}}$ C. Biino, ${ }^{66 \mathrm{a}} \mathrm{C}$. Botta,${ }^{66 \mathrm{a}, 66 \mathrm{~b}}$ N. Cartiglia, ${ }^{66 \mathrm{a}}$ M. Costa ${ }^{66 a, 66 b}$ N. Demaria, ${ }^{66 a}$ A. Graziano, ${ }^{66 a, 66 b}$ C. Mariotti, ${ }^{66 a, f}$ S. Maselli, ${ }^{66 a}$ E. Migliore, ${ }^{66 a, 66 b}$ V. Monaco, ${ }^{66 a, 66 b}$ M. Musich, ${ }^{66 a, f}$ M. M. Obertino, ${ }^{66 a, 66 c}$ N. Pastrone, ${ }^{66 a}$ M. Pelliccioni ${ }^{66 a}$ A. Potenza, ${ }^{66 a, 66 b}$ A. Romero, ${ }^{66 a, 66 b}$ M. Ruspa, ${ }^{66 a, 66 c}$ R. Sacchi, ${ }^{66 a, 66 b}$ V. Sola, ${ }^{66 a, 66 b}$ A. Solano, ${ }^{66 a, 66 b}$ A. Staiano, ${ }^{66 a}$

A. Vilela Pereira, ${ }^{66 a}$ S. Belforte, ${ }^{67 a}$ F. Cossutti, ${ }^{67 a}$ G. Della Ricca, ${ }^{67 a, 67 b}$ B. Gobbo, ${ }^{67 a}$ M. Marone, ${ }^{67 a, 67 b, f}$ D. Montanino, ${ }^{67 \mathrm{a}, 67 \mathrm{~b}, \mathrm{f}}$ A. Penzo, ${ }^{67 \mathrm{a}}$ A. Schizzi, ${ }^{67 \mathrm{a}, 67 \mathrm{~b}}$ S. G. Heo ${ }^{68}$ T. Y. Kim, ${ }^{68}$ S. K. Nam, ${ }^{68}$ S. Chang, ${ }^{69}$ J. Chung, ${ }^{69}$ D. H. Kim, ${ }^{69}$ G. N. Kim, ${ }^{69}$ D. J. Kong, ${ }^{69}$ H. Park, ${ }^{69}$ S. R. Ro, ${ }^{69}$ D. C. Son, ${ }^{69}$ T. Son, ${ }^{69}$ J. Y. Kim, ${ }^{70}$ Zero J. Kim, ${ }^{70}$ S. Song, ${ }^{70}$ H. Y. Jo, ${ }^{71}$ S. Choi, ${ }^{72}$ D. Gyun, ${ }^{72}$ B. Hong, ${ }^{72}$ M. Jo, ${ }^{72}$ H. Kim, ${ }^{72}$ T. J. Kim, ${ }^{72}$ K. S. Lee, ${ }^{72}$ D. H. Moon, ${ }^{72}$ S. K. Park ${ }^{72}$ E. Seo,${ }^{72}$ M. Choi ${ }^{73}$ S. Kang,${ }^{73}$ H. Kim,${ }^{73}$ J. H. Kim,${ }^{73}$ C. Park ${ }^{73}$ I. C. Park, ${ }^{73}$ S. Park,${ }^{73}$ G. Ryu, ${ }^{73}$ Y. Cho, ${ }^{74}$ Y. Choi ${ }^{74}$ Y. K. Choi, ${ }^{74}$ J. Goh, ${ }^{74}$ M. S. Kim,${ }^{74}$ E. Kwon,${ }^{74}$ B. Lee,${ }^{74}$ J. Lee, ${ }^{74}$ S. Lee,${ }^{74}$ H. Seo, ${ }^{74}$ I. Yu, ${ }^{74}$ M. J. Bilinskas, ${ }^{75}$ I. Grigelionis, ${ }^{75}$ M. Janulis, ${ }^{75}$ A. Juodagalvis, ${ }^{75}$ H. Castilla-Valdez, ${ }^{76}$ E. De La Cruz-Burelo, ${ }^{76}$ I. Heredia-de La Cruz,${ }^{76}$ R. Lopez-Fernandez, ${ }^{76}$ R. Magaña Villalba, ${ }^{76}$ J. Martínez-Ortega, ${ }^{76}$

A. Sánchez-Hernández, ${ }^{76}$ L. M. Villasenor-Cendejas, ${ }^{76}$ S. Carrillo Moreno, ${ }^{77}$ F. Vazquez Valencia, ${ }^{77}$ H. A. Salazar Ibarguen, ${ }^{78}$ E. Casimiro Linares, ${ }^{79}$ A. Morelos Pineda,${ }^{79}$ M. A. Reyes-Santos, ${ }^{79}$ D. Krofcheck,${ }^{80}$ A. J. Bell, ${ }^{81}$ P. H. Butler, ${ }^{81}$ R. Doesburg, ${ }^{81}$ S. Reucroft,${ }^{81}$ H. Silverwood, ${ }^{81}$ M. Ahmad, ${ }^{82}$ M. I. Asghar, ${ }^{82}$ H. R. Hoorani, ${ }^{82}$ S. Khalid, ${ }^{82}$ W. A. Khan, ${ }^{82}$ T. Khurshid, ${ }^{82}$ S. Qazi, ${ }^{82}$ M. A. Shah, ${ }^{82}$ M. Shoaib, ${ }^{82}$ G. Brona, ${ }^{83}$ K. Bunkowski, ${ }^{83}$ M. Cwiok, ${ }^{83}$ W. Dominik, ${ }^{83}$ K. Doroba, ${ }^{83}$ A. Kalinowski, ${ }^{83}$ M. Konecki, ${ }^{83}$ J. Krolikowski, ${ }^{83}$ H. Bialkowska, ${ }^{84}$ B. Boimska ${ }^{84}$ T. Frueboes, ${ }^{84}$ R. Gokieli ${ }^{84}$ M. Górski, ${ }^{84}$ M. Kazana ${ }^{84}$ K. Nawrocki,${ }^{84}$

K. Romanowska-Rybinska, ${ }^{84}$ M. Szleper, ${ }^{84}$ G. Wrochna ${ }^{84}$ P. Zalewski, ${ }^{84}$ N. Almeida ${ }^{85}$ P. Bargassa, ${ }^{85}$ A. David ${ }^{85}$ P. Faccioli ${ }^{85}$ M. Fernandes, ${ }^{85}$ P. G. Ferreira Parracho,${ }^{85}$ M. Gallinaro, ${ }^{85}$ J. Seixas,${ }^{85}$ J. Varela, ${ }^{85}$ P. Vischia, ${ }^{85}$ I. Belotelov, ${ }^{86}$ P. Bunin, ${ }^{86}$ M. Gavrilenko, ${ }^{86}$ I. Golutvin, ${ }^{86}$ I. Gorbunov, ${ }^{86}$ A. Kamenev, ${ }^{86}$ V. Karjavin, ${ }^{86}$ G. Kozlov, ${ }^{86}$ 
A. Lanev, ${ }^{86}$ A. Malakhov, ${ }^{86}$ P. Moisenz, ${ }^{86}$ V. Palichik, ${ }^{86}$ V. Perelygin, ${ }^{86}$ S. Shmatov, ${ }^{86}$ V. Smirnov, ${ }^{86}$ A. Volodko, ${ }^{86}$ A. Zarubin, ${ }^{86}$ S. Evstyukhin, ${ }^{87}$ V. Golovtsov, ${ }^{87}$ Y. Ivanov, ${ }^{87}$ V. Kim,${ }^{87}$ P. Levchenko, ${ }^{87}$ V. Murzin, ${ }^{87}$ V. Oreshkin, ${ }^{87}$ I. Smirnov, ${ }^{87}$ V. Sulimov, ${ }^{87}$ L. Uvarov, ${ }^{87}$ S. Vavilov, ${ }^{87}$ A. Vorobyev,${ }^{87}$ An. Vorobyev, ${ }^{87}$ Yu. Andreev, ${ }^{88}$ A. Dermenev ${ }^{88}$ S. Gninenko, ${ }^{88}$ N. Golubev, ${ }^{88}$ M. Kirsanov, ${ }^{88}$ N. Krasnikov, ${ }^{88}$ V. Matveev, ${ }^{88}$ A. Pashenkov, ${ }^{88}$ D. Tlisov ${ }^{88}$ A. Toropin ${ }^{88}$ V. Epshteyn, ${ }^{89}$ M. Erofeeva, ${ }^{89}$ V. Gavrilov,${ }^{89}$ M. Kossov, ${ }^{89, f}$ N. Lychkovskaya ${ }^{89}$ V. Popov, ${ }^{89}$ G. Safronov, ${ }^{89}$ S. Semenov, ${ }^{89}$ V. Stolin, ${ }^{89}$ E. Vlasov, ${ }^{89}$ A. Zhokin, ${ }^{89}$ A. Belyaev,${ }^{90}$ E. Boos, ${ }^{90}$ M. Dubinin, ${ }^{90, \mathrm{e}}$ L. Dudko, ${ }^{90}$ A. Ershov, ${ }^{90}$ A. Gribushin, ${ }^{90}$ V. Klyukhin, ${ }^{90}$ O. Kodolova, ${ }^{90}$ I. Lokhtin, ${ }^{90}$ A. Markina, ${ }^{90}$ S. Obraztsov, ${ }^{90}$ M. Perfilov, ${ }^{90}$ S. Petrushanko, ${ }^{90}$ A. Popov,${ }^{90}$ L. Sarycheva, ${ }^{90, a}$ V. Savrin,${ }^{90}$ A. Snigirev,${ }^{90}$ V. Andreev, ${ }^{91}$ M. Azarkin, ${ }^{91}$ I. Dremin, ${ }^{91}$ M. Kirakosyan, ${ }^{91}$ A. Leonidov,${ }^{91}$ G. Mesyats, ${ }^{91}$ S. V. Rusakov, ${ }^{91}$ A. Vinogradov, ${ }^{91}$ I. Azhgirey, ${ }^{92}$ I. Bayshev,${ }^{92}$ S. Bitioukov, ${ }^{92}$ V. Grishin, ${ }^{92, f}$ V. Kachanov, ${ }^{92}$ D. Konstantinov, ${ }^{92}$ A. Korablev, ${ }^{92}$ V. Krychkine, ${ }^{92}$ V. Petrov,${ }^{92}$ R. Ryutin, ${ }^{92}$ A. Sobol,${ }^{92}$ L. Tourtchanovitch, ${ }^{92}$ S. Troshin, ${ }^{92}$ N. Tyurin, ${ }^{92}$ A. Uzunian, ${ }^{92}$ A. Volkov, ${ }^{92}$ P. Adzic, ${ }^{93, d d}$ M. Djordjevic, ${ }^{93}$ M. Ekmedzic,${ }^{93}$ D. Krpic, ${ }^{93, d d}$ J. Milosevic, ${ }^{93}$ M. Aguilar-Benitez,${ }^{94}$ J. Alcaraz Maestre,${ }^{94}$ P. Arce,${ }^{94}$ C. Battilana,${ }^{94}$ E. Calvo,${ }^{94}$ M. Cerrada, ${ }^{94}$ M. Chamizo Llatas, ${ }^{94}$ N. Colino, ${ }^{94}$ B. De La Cruz, ${ }^{94}$ A. Delgado Peris, ${ }^{94}$ C. Diez Pardos, ${ }^{94}$ D. Domínguez Vázquez,${ }^{94}$ C. Fernandez Bedoya,${ }^{94}$ J. P. Fernández Ramos, ${ }^{94}$ A. Ferrando, ${ }^{94}$ J. Flix,${ }^{94}$ M. C. Fouz, ${ }^{94}$ P. Garcia-Abia,${ }^{94}$

O. Gonzalez Lopez,${ }^{94}$ S. Goy Lopez, ${ }^{94}$ J. M. Hernandez, ${ }^{94}$ M. I. Josa, ${ }^{94}$ G. Merino, ${ }^{94}$ J. Puerta Pelayo, ${ }^{94}$

A. Quintario Olmeda, ${ }^{94}$ I. Redondo, ${ }^{94}$ L. Romero, ${ }^{94}$ J. Santaolalla, ${ }^{94}$ M. S. Soares,${ }^{94}$ C. Willmott,${ }^{94}$ C. Albajar, ${ }^{95}$ G. Codispoti, ${ }^{95}$ J.F. de Trocóniz, ${ }^{95}$ J. Cuevas, ${ }^{96}$ J. Fernandez Menendez, ${ }^{96}$ S. Folgueras, ${ }^{96}$ I. Gonzalez Caballero, ${ }^{96}$ L. Lloret Iglesias, ${ }^{96}$ J. Piedra Gomez, ${ }^{96, e e}$ J. A. Brochero Cifuentes, ${ }^{97}$ I. J. Cabrillo, ${ }^{97}$ A. Calderon, ${ }^{97}$ S. H. Chuang, ${ }^{97}$

J. Duarte Campderros, ${ }^{97}$ M. Felcini, ${ }^{97, f f}$ M. Fernandez, ${ }^{97}$ G. Gomez, ${ }^{97}$ J. Gonzalez Sanchez, ${ }^{97}$ C. Jorda, ${ }^{97}$

P. Lobelle Pardo, ${ }^{97}$ A. Lopez Virto, ${ }^{97}$ J. Marco,${ }^{97}$ R. Marco,${ }^{97}$ C. Martinez Rivero,${ }^{97}$ F. Matorras, ${ }^{97}$

F. J. Munoz Sanchez, ${ }^{97}$ T. Rodrigo, ${ }^{97}$ A. Y. Rodríguez-Marrero, ${ }^{97}$ A. Ruiz-Jimeno, ${ }^{97}$ L. Scodellaro, ${ }^{97}$ M. Sobron Sanudo, ${ }^{97}$ I. Vila, ${ }^{97}$ R. Vilar Cortabitarte, ${ }^{97}$ D. Abbaneo, ${ }^{98}$ E. Auffray,${ }^{98}$ G. Auzinger,${ }^{98}$ P. Baillon, ${ }^{98}$ A. H. Ball, ${ }^{98}$ D. Barney, ${ }^{98}$ C. Bernet, ${ }^{98,9}$ G. Bianchi, ${ }^{98}$ P. Bloch, ${ }^{98}$ A. Bocci,${ }^{98}$ A. Bonato, ${ }^{98}$ H. Breuker, ${ }^{98}$ T. Camporesi,${ }^{98}$ G. Cerminara, ${ }^{98}$ T. Christiansen, ${ }^{98}$ J. A. Coarasa Perez, ${ }^{98}$ D. D'Enterria, ${ }^{98}$ A. Dabrowski, ${ }^{98}$ A. De Roeck, ${ }^{98}$ S. Di Guida, ${ }^{98}$ M. Dobson, ${ }^{98}$ N. Dupont-Sagorin, ${ }^{98}$ A. Elliott-Peisert, ${ }^{98}$ B. Frisch,${ }^{98}$ W. Funk, ${ }^{98}$ G. Georgiou, ${ }^{98}$ M. Giffels, ${ }^{98}$ D. Gigi,${ }^{98}$ K. Gill,${ }^{98}$ D. Giordano, ${ }^{98}$ M. Giunta,${ }^{98}$ F. Glege,${ }^{98}$

R. Gomez-Reino Garrido, ${ }^{98}$ P. Govoni, ${ }^{98}$ S. Gowdy, ${ }^{98}$ R. Guida, ${ }^{98}$ M. Hansen, ${ }^{98}$ P. Harris, ${ }^{98}$ C. Hartl, ${ }^{98}$ J. Harvey,${ }^{98}$ B. Hegner, ${ }^{98}$ A. Hinzmann, ${ }^{98}$ V. Innocente, ${ }^{98}$ P. Janot,${ }^{98}$ K. Kaadze, ${ }^{98}$ E. Karavakis, ${ }^{98}$ K. Kousouris, ${ }^{98}$ P. Lecoq, ${ }^{98}$ Y.-J. Lee, ${ }^{98}$ P. Lenzi,${ }^{98}$ C. Lourenço, ${ }^{98}$ T. Mäki,${ }^{98}$ M. Malberti,${ }^{98}$ L. Malgeri, ${ }^{98}$ M. Mannelli, ${ }^{98}$ L. Masetti,${ }^{98}$ F. Meijers, ${ }^{98}$ S. Mersi, ${ }^{98}$ E. Meschi,${ }^{98}$ R. Moser,${ }^{98}$ M. U. Mozer, ${ }^{98}$ M. Mulders, ${ }^{98}$ P. Musella, ${ }^{98}$ E. Nesvold,${ }^{98}$ T. Orimoto, ${ }^{98}$ L. Orsini, ${ }^{98}$ E. Palencia Cortezon, ${ }^{98}$ E. Perez, ${ }^{98}$ A. Petrilli, ${ }^{98}$ A. Pfeiffer, ${ }^{98}$ M. Pierini, ${ }^{98}$ M. Pimiä, ${ }^{98}$ D. Piparo, ${ }^{98}$ G. Polese, ${ }^{98}$ L. Quertenmont, ${ }^{98}$ A. Racz,${ }^{98}$ W. Reece, ${ }^{98}$ J. Rodrigues Antunes, ${ }^{98}$ G. Rolandi, ${ }^{98, g g}$ T. Rommerskirchen, ${ }^{98}$ C. Rovelli, ${ }^{98, \text { hh }}$ M. Rovere, ${ }^{98}$ H. Sakulin, ${ }^{98}$ F. Santanastasio, ${ }^{98}$ C. Schäfer, ${ }^{98}$ C. Schwick, ${ }^{98}$ I. Segoni,${ }^{98}$ S. Sekmen, ${ }^{98}$ A. Sharma,${ }^{98}$ P. Siegrist,${ }^{98}$ P. Silva, ${ }^{98}$ M. Simon,${ }^{98}$ P. Sphicas, ${ }^{98, \text { ii }}$ D. Spiga,${ }^{98}$ M. Spiropulu, ${ }^{98, \mathrm{e}}$ M. Stoye,${ }^{98}$ A. Tsirou ${ }^{98}$ G. I. Veres,${ }^{98, r}$ J. R. Vlimant, ${ }^{98}$ H. K. Wöhri, ${ }^{98}$ S. D. Worm, ${ }^{98, j j}$ W. D. Zeuner, ${ }^{98}$ W. Bertl,${ }^{99}$ K. Deiters, ${ }^{99}$ W. Erdmann, ${ }^{99}$ K. Gabathuler, ${ }^{99}$ R. Horisberger,${ }^{99}$ Q. Ingram, ${ }^{99}$ H. C. Kaestlii, ${ }^{99}$ S. König, ${ }^{99}$ D. Kotlinski, ${ }^{99}$ U. Langenegger, ${ }^{99}$ F. Meier, ${ }^{99}$ D. Renker, ${ }^{99}$ T. Rohe, ${ }^{99}$ J. Sibille, ${ }^{99, k k}$ L. Bäni,${ }^{100}$ P. Bortignon, ${ }^{100}$ M. A. Buchmann, ${ }^{100}$ B. Casal, ${ }^{100}$ N. Chanon, ${ }^{100}$ Z. Chen, ${ }^{100}$ A. Deisher, ${ }^{100}$ G. Dissertori, ${ }^{100}$ M. Dittmar, ${ }^{100}$ M. Dünser, ${ }^{100}$ J. Eugster, ${ }^{100}$ K. Freudenreich, ${ }^{100}$ C. Grab,${ }^{100}$ D. Hits, ${ }^{100}$ P. Lecomte, ${ }^{100}$ W. Lustermann, ${ }^{100}$ A. C. Marini,${ }^{100}$ P. Martinez Ruiz del Arbol, ${ }^{100}$ N. Mohr,${ }^{100}$ F. Moortgat,${ }^{100}$ C. Nägeli, ${ }^{100,11}$ P. Nef, ${ }^{100}$ F. Nessi-Tedaldi, ${ }^{100}$ F. Pandolfi, ${ }^{100}$ L. Pape, ${ }^{100}$ F. Pauss, ${ }^{100}$ M. Peruzzi ${ }^{100}$ F. J. Ronga, ${ }^{100}$ M. Rossini, ${ }^{100}$ L. Sala, ${ }^{100}$ A. K. Sanchez, ${ }^{100}$ A. Starodumov, ${ }^{100, \mathrm{~mm}}$ B. Stieger, ${ }^{100}$ M. Takahashi, ${ }^{100}$ L. Tauscher, ${ }^{100, a}$ A. Thea, ${ }^{100}$ K. Theofilatos, ${ }^{100}$ D. Treille, ${ }^{100}$ C. Urscheler, ${ }^{100}$ R. Wallny, ${ }^{100}$ H. A. Weber, ${ }^{100}$ L. Wehrli, ${ }^{100}$ E. Aguilo, ${ }^{101}$ C. Amsler,${ }^{101}$ V. Chiochia,${ }^{101}$ S. De Visscher, ${ }^{101}$ C. Favaro, ${ }^{101}$ M. Ivova Rikova, ${ }^{101}$ B. Millan Mejias, ${ }^{101}$ P. Otiougova, ${ }^{101}$ P. Robmann, ${ }^{101}$ H. Snoek, ${ }^{101}$ S. Tupputi, ${ }^{101}$ M. Verzetti, ${ }^{101}$ Y. H. Chang, ${ }^{102}$ K. H. Chen, ${ }^{102}$ C. M. Kuo ${ }^{102}$ S. W. Li, ${ }^{102}$ W. Lin,${ }^{102}$ Z. K. Liu, ${ }^{102}$ Y. J. Lu, ${ }^{102}$ D. Mekterovic, ${ }^{102}$ A. P. Singh ${ }^{102}$ R. Volpe, ${ }^{102}$ S. S. Yu, ${ }^{102}$ P. Bartalini, ${ }^{103}$ P. Chang, ${ }^{103}$ Y. H. Chang,,${ }^{103}$ Y. W. Chang,,${ }^{103}$ Y. Chao, ${ }^{103}$ K. F. Chen, ${ }^{103}$ C. Dietz, ${ }^{103}$ U. Grundler, ${ }^{103}$ W.-S. Hou, ${ }^{103}$ Y. Hsiung, ${ }^{103}$ K. Y. Kao, ${ }^{103}$ Y. J. Lei, ${ }^{103}$ R.-S. Lu, ${ }^{103}$ D. Majumder, ${ }^{103}$ E. Petrakou, ${ }^{103}$ X. Shi, ${ }^{103}$ J. G. Shiu, ${ }^{103}$ Y. M. Tzeng, ${ }^{103}$ X. Wan, ${ }^{103}$ M. Wang, ${ }^{103}$ A. Adiguzel, ${ }^{104}$ M. N. Bakirci, ${ }^{104, n n}$ S. Cerci, ${ }^{104, o 0}$ C. Dozen, ${ }^{104}$ I. Dumanoglu, ${ }^{104}$ E. Eskut, ${ }^{104}$ S. Girgis,${ }^{104}$ G. Gokbulut, ${ }^{104}$ E. Gurpinar, ${ }^{104}$ I. Hos, ${ }^{104}$ E. E. Kangal,${ }^{104}$ 
G. Karapinar, ${ }^{104}$ A. Kayis Topaksu, ${ }^{104}$ G. Onengut ${ }^{104}$ K. Ozdemir, ${ }^{104}$ S. Ozturk, ${ }^{104, p p}$ A. Polatoz, ${ }^{104}$ K. Sogut, ${ }^{104, q q}$ D. Sunar Cerci, ${ }^{104, \text { oo }}$ B. Tali, ${ }^{104, \text { oo }}$ H. Topakli, ${ }^{104, \text { nn }}$ L. N. Vergili, ${ }^{104}$ M. Vergili, ${ }^{104}$ I. V. Akin, ${ }^{105}$ T. Aliev, ${ }^{105}$ B. Bilin, ${ }^{105}$ S. Bilmis, ${ }^{105}$ M. Deniz, ${ }^{105}$ H. Gamsizkan, ${ }^{105}$ A. M. Guler, ${ }^{105}$ K. Ocalan, ${ }^{105}$ A. Ozpineci, ${ }^{105}$ M. Serin,${ }^{105}$ R. Sever, ${ }^{105}$ U. E. Surat, ${ }^{105}$ M. Yalvac, ${ }^{105}$ E. Yildirim, ${ }^{105}$ M. Zeyrek, ${ }^{105}$ E. Gülmez, ${ }^{106}$ B. Isildak, ${ }^{106, r r}$ M. Kaya, ${ }^{106, s s}$ O. Kaya, ${ }^{106, s s}$ S. Ozkorucuklu, ${ }^{106, \text { tt }}$ N. Sonmez, ${ }^{106, \text { uu }}$ K. Cankocak, ${ }^{107}$ L. Levchuk, ${ }^{108}$ F. Bostock, ${ }^{109}$ J. J. Brooke, ${ }^{109}$ E. Clement ${ }^{109}$ D. Cussans,${ }^{109}$ H. Flacher ${ }^{109}$ R. Frazier, ${ }^{109}$ J. Goldstein, ${ }^{109}$ M. Grimes, ${ }^{109}$ G. P. Heath, ${ }^{109}$ H. F. Heath, ${ }^{109}$ L. Kreczko, ${ }^{109}$ S. Metson, ${ }^{109}$ D. M. Newbold, ${ }^{109, j j}$ K. Nirunpong, ${ }^{109}$ A. Poll, ${ }^{109}$ S. Senkin, ${ }^{109}$ V. J. Smith, ${ }^{109}$ T. Williams, ${ }^{109}$ L. Basso, ${ }^{110, v v}$ K. W. Bell, ${ }^{110}$ A. Belyaev, ${ }^{110, v v}$ C. Brew, ${ }^{110}$ R. M. Brown,,${ }^{110}$ D. J. A. Cockerill, ${ }^{110}$ J. A. Coughlan, ${ }^{110}$ K. Harder,${ }^{110}$ S. Harper, ${ }^{110}$ J. Jackson, ${ }^{110}$ B. W. Kennedy, ${ }^{110}$ E. Olaiya, ${ }^{110}$ D. Petyt,${ }^{110}$ B. C. Radburn-Smith, ${ }^{110}$ C. H. Shepherd-Themistocleous, ${ }^{110}$ I. R. Tomalin, ${ }^{110}$ W. J. Womersley, ${ }^{110}$ R. Bainbridge, ${ }^{111}$ G. Ball, ${ }^{111}$ R. Beuselinck, ${ }^{111}$ O. Buchmuller, ${ }^{111}$ D. Colling, ${ }^{111}$ N. Cripps, ${ }^{111}$ M. Cutajar, ${ }^{111}$ P. Dauncey, ${ }^{111}$ G. Davies, ${ }^{111}$ M. Della Negra, ${ }^{111}$ W. Ferguson, ${ }^{111}$ J. Fulcher, ${ }^{111}$ D. Futyan, ${ }^{111}$ A. Gilbert, ${ }^{111}$ A. Guneratne Bryer, ${ }^{111}$ G. Hall, ${ }^{111}$ Z. Hatherell, ${ }^{111}$ J. Hays, ${ }^{111}$ G. Iles, ${ }^{111}$ M. Jarvis, ${ }^{111}$ G. Karapostoli, ${ }^{111}$ L. Lyons, ${ }^{111}$ A.-M. Magnan, ${ }^{111}$ J. Marrouche, ${ }^{111}$ B. Mathias,${ }^{111}$ R. Nandi, ${ }^{111}$ J. Nash, ${ }^{111}$ A. Nikitenko, ${ }^{111, m m}$ A. Papageorgiou, ${ }^{111}$ J. Pela, ${ }^{111, f}$ M. Pesaresi,${ }^{111}$ K. Petridis, ${ }^{111}$ M. Pioppi, ${ }^{111, w w}$ D. M. Raymond, ${ }^{111}$ S. Rogerson, ${ }^{111}$ A. Rose, ${ }^{111}$ M. J. Ryan, ${ }^{111}$ C. Seez, ${ }^{111}$ P. Sharp, ${ }^{111, a}$ A. Sparrow, ${ }^{111}$ A. Tapper, ${ }^{111}$ M. Vazquez Acosta, ${ }^{111}$ T. Virdee,${ }^{111}$ S. Wakefield, ${ }^{111}$ N. Wardle, ${ }^{111}$ T. Whyntie, ${ }^{111}$ M. Chadwick, ${ }^{112}$ J. E. Cole,${ }^{112}$ P. R. Hobson, ${ }^{112}$ A. Khan, ${ }^{112}$ P. Kyberd, ${ }^{112}$ D. Leggat, ${ }^{112}$ D. Leslie, ${ }^{112}$ W. Martin,${ }^{112}$ I. D. Reid, ${ }^{112}$ P. Symonds, ${ }^{112}$ L. Teodorescu, ${ }^{112}$ M. Turner, ${ }^{112}$ K. Hatakeyama, ${ }^{113}$ H. Liu, ${ }^{113}$ T. Scarborough, ${ }^{113}$ C. Henderson, ${ }^{114}$ P. Rumerio, ${ }^{114}$ A. Avetisyan, ${ }^{115}$ T. Bose,${ }^{115}$ C. Fantasia, ${ }^{115}$ A. Heister, ${ }^{115}$ J. St. John, ${ }^{115}$ P. Lawson, ${ }^{115}$ D. Lazic, ${ }^{115}$ J. Rohlf, ${ }^{115}$ D. Sperka, ${ }^{115}$ L. Sulak, ${ }^{115}$ J. Alimena, ${ }^{116}$ S. Bhattacharya, ${ }^{116}$ D. Cutts, ${ }^{116}$ A. Ferapontov, ${ }^{116}$ U. Heintz, ${ }^{116}$ S. Jabeen, ${ }^{116}$ G. Kukartsev, ${ }^{116}$ E. Laird ${ }^{116}$ G. Landsberg, ${ }^{116}$ M. Luk, ${ }^{116}$ M. Narain, ${ }^{116}$ D. Nguyen, ${ }^{116}$ M. Segala, ${ }^{116}$ T. Sinthuprasith, ${ }^{116}$ T. Speer, ${ }^{116}$ K. V. Tsang, ${ }^{116}$ R. Breedon, ${ }^{117}$ G. Breto, ${ }^{117}$ M. Calderon De La Barca Sanchez, ${ }^{117}$ S. Chauhan, ${ }^{117}$ M. Chertok, ${ }^{117}$ J. Conway, ${ }^{117}$ R. Conway, ${ }^{117}$ P. T. Cox,${ }^{117}$ J. Dolen, ${ }^{117}$ R. Erbacher, ${ }^{117}$ M. Gardner, ${ }^{117}$ R. Houtz, ${ }^{117}$ W. Ko, ${ }^{117}$ A. Kopecky, ${ }_{17}^{17}$ R. Lander, ${ }^{17}$ O. Mall, ${ }^{117}$ T. Miceli, ${ }^{117}$ R. Nelson, ${ }^{117}$ D. Pellett, ${ }^{117}$ B. Rutherford,${ }^{117}$ M. Searle, ${ }^{117}$ J. Smith, ${ }^{117}$ M. Squires, ${ }^{117}$ M. Tripathi, ${ }^{117}$ R. Vasquez Sierra, ${ }^{117}$ V. Andreev, ${ }^{118}$ D. Cline, ${ }^{118}$ R. Cousins, ${ }^{18}$ J. Duris, ${ }^{118}$ S. Erhan, ${ }^{118}$ P. Everaerts, ${ }^{118}$ C. Farrell, ${ }^{118}$ J. Hauser,${ }^{118}$ M. Ignatenko, ${ }^{118}$ C. Jarvis,${ }^{118}$ C. Plager, ${ }^{118}$ G. Rakness, ${ }^{118}$ P. Schlein, ${ }^{118, a}$ J. Tucker, ${ }^{18}$ V. Valuev, ${ }^{118}$ M. Weber, ${ }^{18}$ J. Babb,${ }^{119}$ R. Clare, ${ }^{119}$ M. E. Dinardo, ${ }^{119}$ J. Ellison, ${ }^{119}$ J. W. Gary, ${ }^{119}$ F. Giordano, ${ }^{119}$ G. Hanson, ${ }^{119}$ G. Y. Jeng, ${ }^{119, x x}$ H. Liu, ${ }^{119}$ O. R. Long, ${ }^{119}$ A. Luthra, ${ }^{119}$ H. Nguyen, ${ }^{119}$ S. Paramesvaran, ${ }^{119}$ J. Sturdy,${ }^{119}$ S. Sumowidagdo, ${ }^{119}$ R. Wilken, ${ }^{119}$ S. Wimpenny, ${ }^{119}$ W. Andrews, ${ }^{120}$ J. G. Branson, ${ }^{120}$ G. B. Cerati, ${ }^{120}$ S. Cittolin, ${ }^{120}$ D. Evans, ${ }^{120}$ F. Golf, ${ }^{120}$ A. Holzner, ${ }^{120}$ R. Kelley, ${ }^{120}$

M. Lebourgeois, ${ }^{120}$ J. Letts, ${ }^{120}$ I. Macneill, ${ }^{120}$ B. Mangano, ${ }^{120}$ S. Padhi, ${ }^{120}$ C. Palmer, ${ }^{120}$ G. Petrucciani, ${ }^{120}$ M. Pieri, ${ }^{120}$ M. Sani, ${ }^{120}$ V. Sharma,${ }^{120}$ S. Simon, ${ }^{120}$ E. Sudano, ${ }^{120}$ M. Tadel, ${ }^{120}$ Y. Tu, ${ }^{120}$ A. Vartak,,${ }^{120}$ S. Wasserbaech, ${ }^{120, \text { yy }}$ F. Würthwein, ${ }^{120}$ A. Yagil, ${ }^{120}$ J. Yoo, ${ }^{120}$ D. Barge,${ }^{121}$ R. Bellan, ${ }^{121}$ C. Campagnari, ${ }^{121}$ M. D’Alfonso, ${ }^{121}$ T. Danielson, ${ }^{121}$ K. Flowers, ${ }^{121}$ P. Geffert, ${ }^{121}$ J. Incandela,${ }^{121}$ C. Justus, ${ }^{121}$ P. Kalavase,${ }^{121}$ S. A. Koay, ${ }^{121}$ D. Kovalskyi, ${ }^{121}$ V. Krutelyov, ${ }^{121}$ S. Lowette, ${ }^{121}$ N. Mccoll, ${ }^{121}$ V. Pavlunin, ${ }^{121}$ F. Rebassoo, ${ }^{121}$ J. Ribnik, ${ }^{121}$ J. Richman, ${ }^{121}$ R. Rossin, ${ }^{121}$ D. Stuart, ${ }^{121}$ W. To, ${ }^{121}$ C. West,${ }^{121}$ A. Apresyan, ${ }^{122}$ A. Bornheim, ${ }^{122}$ Y. Chen, ${ }^{122}$ E. Di Marco, ${ }^{122}$ J. Duarte, ${ }^{122}$ M. Gataullin, ${ }^{122}$ Y. Ma, ${ }^{122}$ A. Mott,,${ }^{122}$ H. B. Newman, ${ }^{122}$ C. Rogan, ${ }^{122}$ V. Timciuc, ${ }^{122}$ P. Traczyk, ${ }^{122}$ J. Veverka, ${ }^{122}$ R. Wilkinson, ${ }^{122}$ Y. Yang, ${ }^{122}$ R. Y. Zhu, ${ }^{122}$ B. Akgun, ${ }^{123}$ R. Carroll, ${ }^{123}$ T. Ferguson, ${ }^{123}$ Y. Iiyama, ${ }^{123}$ D. W. Jang, ${ }^{123}$ Y. F. Liu, ${ }^{123}$ M. Paulini, ${ }^{123}$ H. Vogel, ${ }^{123}$ I. Vorobiev, ${ }^{123}$ J. P. Cumalat ${ }^{124}$ B. R. Drell, ${ }^{124}$ C. J. Edelmaier, ${ }^{124}$ W. T. Ford ${ }^{124}$ A. Gaz,${ }^{124}$ B. Heyburn, ${ }^{124}$ E. Luiggi Lopez, ${ }^{124}$ J. G. Smith, ${ }^{124}$ K. Stenson, ${ }^{124}$ K. A. Ulmer, ${ }^{124}$ S. R. Wagner, ${ }^{124}$ J. Alexander, ${ }^{125}$ A. Chatterjee, ${ }^{125}$ N. Eggert, ${ }^{125}$ L. K. Gibbons, ${ }^{125}$ B. Heltsley, ${ }^{125}$ A. Khukhunaishvili, ${ }^{125}$ B. Kreis, ${ }^{125}$ N. Mirman, ${ }^{125}$ G. Nicolas Kaufman, ${ }^{125}$ J. R. Patterson, ${ }^{125}$ A. Ryd,${ }^{125}$ E. Salvati, ${ }^{125}$ W. Sun, ${ }^{125}$ W. D. Teo, ${ }^{125}$ J. Thom, ${ }^{125}$ J. Thompson, ${ }^{125}$ J. Vaughan, ${ }^{125}$ Y. Weng, ${ }^{125}$ L. Winstrom, ${ }^{125}$ P. Wittich, ${ }^{125}$ D. Winn, ${ }^{126}$ S. Abdullin, ${ }^{127}$ M. Albrow, ${ }^{127}$ J. Anderson, ${ }^{127}$ L. A. T. Bauerdick, ${ }^{127}$ A. Beretvas, ${ }^{127}$ J. Berryhill, ${ }^{127}$ P. C. Bhat, ${ }^{127}$ I. Bloch, ${ }^{127}$ K. Burkett,${ }^{127}$ J. N. Butler, ${ }^{127}$ V. Chetluru, ${ }^{127}$ H. W. K. Cheung, ${ }^{127}$ F. Chlebana, ${ }^{127}$ V. D. Elvira, ${ }^{127}$ I. Fisk, ${ }^{127}$ J. Freeman, ${ }^{127}$ Y. Gao, ${ }^{127}$ D. Green, ${ }^{127}$ O. Gutsche,${ }^{127}$ A. Hahn, ${ }^{127}$ J. Hanlon, ${ }^{127}$ R. M. Harris, ${ }^{127}$ J. Hirschauer, ${ }^{127}$ B. Hooberman, ${ }^{127}$ S. Jindariani, ${ }^{127}$ M. Johnson, ${ }^{127}$ U. Joshi, ${ }^{127}$ B. Kilminster, ${ }^{127}$ B. Klima, ${ }^{127}$ S. Kunori, ${ }^{127}$ S. Kwan, ${ }^{127}$ C. Leonidopoulos, ${ }^{127}$ D. Lincoln, ${ }^{127}$ R. Lipton, ${ }^{127}$ L. Lueking, ${ }^{127}$ J. Lykken, ${ }^{127}$ K. Maeshima, ${ }^{127}$ J. M. Marraffino, ${ }^{127}$ S. Maruyama, ${ }^{127}$ D. Mason, ${ }^{127}$ P. McBride,${ }^{127}$ K. Mishra, ${ }^{127}$ S. Mrenna, ${ }^{127}$ Y. Musienko, ${ }^{127, z z}$ C. Newman-Holmes, ${ }^{127}$ V. O’Dell, ${ }^{127}$ 
O. Prokofyev, ${ }^{127}$ E. Sexton-Kennedy, ${ }^{127}$ S. Sharma, ${ }^{127}$ W. J. Spalding,,${ }^{127}$ L. Spiegel, ${ }^{127}$ P. Tan, ${ }^{127}$ L. Taylor, ${ }^{127}$ S. Tkaczyk, ${ }^{127}$ N. V. Tran, ${ }^{127}$ L. Uplegger, ${ }^{127}$ E. W. Vaandering, ${ }^{127}$ R. Vidal,${ }^{127}$ J. Whitmore, ${ }^{127}$ W. Wu, ${ }^{127}$ F. Yang, ${ }^{127}$ F. Yumiceva, ${ }^{127}$ J. C. Yun, ${ }^{127}$ D. Acosta, ${ }^{128}$ P. Avery, ${ }^{128}$ D. Bourilkov, ${ }^{128}$ M. Chen, ${ }^{128}$ S. Das, ${ }^{128}$ M. De Gruttola, ${ }^{128}$ G. P. Di Giovanni, ${ }^{128}$ D. Dobur, ${ }^{128}$ A. Drozdetskiy, ${ }^{128}$ R. D. Field, ${ }^{128}$ M. Fisher, ${ }^{128}$ Y. Fu, ${ }^{128}$

I. K. Furic, ${ }^{128}$ J. Gartner, ${ }^{128}$ J. Hugon, ${ }^{128}$ B. Kim, ${ }^{128}$ J. Konigsberg, ${ }^{128}$ A. Korytov, ${ }^{128}$ A. Kropivnitskaya, ${ }^{128}$ T. Kypreos, ${ }^{128}$ J. F. Low, ${ }^{128}$ K. Matchev, ${ }^{128}$ P. Milenovic, ${ }^{128, \text { aaa }}$ G. Mitselmakher, ${ }^{128}$ L. Muniz, ${ }^{128}$ R. Remington, ${ }^{128}$ A. Rinkevicius, ${ }^{128}$ P. Sellers, ${ }^{128}$ N. Skhirtladze, ${ }^{128}$ M. Snowball, ${ }^{128}$ J. Yelton, ${ }^{128}$ M. Zakaria, ${ }^{128}$ V. Gaultney, ${ }^{129}$ L. M. Lebolo, ${ }^{129}$ S. Linn, ${ }^{129}$ P. Markowitz, ${ }^{129}$ G. Martinez, ${ }^{129}$ J. L. Rodriguez, ${ }^{129}$ J. R. Adams, ${ }^{130}$ T. Adams, ${ }^{130}$ A. Askew, ${ }^{130}$ J. Bochenek, ${ }^{130}$ J. Chen, ${ }^{130}$ B. Diamond, ${ }^{130}$ S. V. Gleyzer, ${ }^{130}$ J. Haas, ${ }^{130}$ S. Hagopian, ${ }^{130}$ V. Hagopian, ${ }^{130}$ M. Jenkins, ${ }^{130}$ K. F. Johnson, ${ }^{130}$ H. Prosper,${ }^{130}$ V. Veeraraghavan, ${ }^{130}$ M. Weinberg, ${ }^{130}$ M. M. Baarmand, ${ }^{131}$ B. Dorney, ${ }^{131}$ M. Hohlmann, ${ }^{131}$ H. Kalakhety, ${ }^{131}$ I. Vodopiyanov, ${ }^{131}$ M. R. Adams, ${ }^{132}$ I. M. Anghel, ${ }^{132}$ L. Apanasevich, ${ }^{132}$ Y. Bai, ${ }^{132}$ V. E. Bazterra, ${ }^{132}$ R. R. Betts ${ }^{132}$ I. Bucinskaite,${ }^{132}$ J. Callner, ${ }^{132}$ R. Cavanaugh, ${ }^{132}$ C. Dragoiu, ${ }^{132}$ O. Evdokimov, ${ }^{132}$ L. Gauthier, ${ }^{132}$ C. E. Gerber, ${ }^{132}$ S. Hamdan, ${ }^{132}$ D. J. Hofman, ${ }^{132}$ S. Khalatyan, ${ }^{132}$ F. Lacroix,${ }^{132}$ M. Malek, ${ }^{132}$ C. O’Brien, ${ }^{132}$ C. Silkworth, ${ }^{132}$ D. Strom, ${ }^{132}$ N. Varelas, ${ }^{132}$ U. Akgun, ${ }^{133}$ E. A. Albayrak, ${ }^{133}$ B. Bilki, ${ }^{133, \text { bbb }}$ W. Clarida, ${ }^{133}$ F. Duru, ${ }^{133}$ S. Griffiths, ${ }^{133}$ J.-P. Merlo, ${ }^{133}$ H. Mermerkaya, ${ }^{133, \mathrm{ccc}}$ A. Mestvirishvili, ${ }^{133}$ A. Moeller, ${ }^{133}$ J. Nachtman, ${ }^{133}$ C. R. Newsom, ${ }^{133}$ E. Norbeck, ${ }^{133}$ Y. Onel,,${ }^{133}$ F. Ozok,${ }^{133}$ S. Sen, ${ }^{133}$ E. Tiras, ${ }^{133}$ J. Wetzel, ${ }^{133}$ T. Yetkin, ${ }^{133}$ K. Yi, ${ }^{133}$ B. A. Barnett, ${ }^{134}$ B. Blumenfeld, ${ }^{134}$ S. Bolognesi, ${ }^{134}$ D. Fehling, ${ }^{134}$ G. Giurgiu, ${ }^{134}$ A. V. Gritsan, ${ }^{134}$ Z. J. Guo,${ }^{134}$ G. Hu, ${ }^{134}$ P. Maksimovic,${ }^{134}$ S. Rappoccio, ${ }^{134}$ M. Swartz, ${ }^{134}$ A. Whitbeck, ${ }^{134}$ P. Baringer, ${ }^{135}$ A. Bean,${ }^{135}$ G. Benelli, ${ }^{135}$ O. Grachov, ${ }^{135}$ R. P. Kenny Iii, ${ }^{135}$ M. Murray, ${ }^{135}$ D. Noonan, ${ }^{135}$ S. Sanders, ${ }^{135}$ R. Stringer,,${ }^{135}$ G. Tinti, ${ }^{135}$ J. S. Wood, ${ }^{135}$ V. Zhukova, ${ }^{135}$ A. F. Barfuss, ${ }^{136}$ T. Bolton, ${ }^{136}$ I. Chakaberia, ${ }^{136}$ A. Ivanov, ${ }^{136}$ S. Khalil, ${ }^{136}$ M. Makouski, ${ }^{136}$ Y. Maravin, ${ }^{136}$ S. Shrestha, ${ }^{136}$ I. Svintradze, ${ }^{136}$ J. Gronberg, ${ }^{137}$ D. Lange,${ }^{137}$ D. Wright,${ }^{137}$ A. Baden, ${ }^{138}$ M. Boutemeur, ${ }^{138}$ B. Calvert, ${ }^{138}$ S. C. Eno, ${ }^{138}$ J. A. Gomez, ${ }^{138}$ N. J. Hadley ${ }^{138}$ R. G. Kellogg, ${ }^{138}$ M. Kirn, ${ }^{138}$ T. Kolberg, ${ }^{138}$ Y. Lu, ${ }^{138}$ M. Marionneau, ${ }^{138}$ A. C. Mignerey, ${ }^{138}$ K. Pedro, ${ }^{138}$ A. Peterman, ${ }^{138}$ A. Skuja, ${ }^{138}$ J. Temple, ${ }^{138}$ M. B. Tonjes, ${ }^{138}$ S. C. Tonwar, ${ }^{138}$ E. Twedt, ${ }^{138}$ G. Bauer, ${ }^{139}$ J. Bendavid, ${ }^{139}$ W. Busza, ${ }^{139}$ E. Butz, ${ }^{139}$ I. A. Cali, ${ }^{139}$ M. Chan, ${ }^{139}$ V. Dutta, ${ }^{139}$ G. Gomez Ceballos, ${ }^{139}$ M. Goncharov, ${ }^{139}$ K. A. Hahn, ${ }^{139}$ Y. Kim, ${ }^{139}$ M. Klute, ${ }^{139}$ W. Li, ${ }^{139}$ P. D. Luckey, ${ }^{139}$ T. Ma, ${ }^{139}$ S. Nahn, ${ }^{139}$ C. Paus, ${ }^{139}$ D. Ralph, ${ }^{139}$ C. Roland, ${ }^{139}$ G. Roland,${ }^{139}$ M. Rudolph, ${ }^{139}$ G. S. F. Stephans, ${ }^{139}$ F. Stöckli, ${ }^{139}$ K. Sumorok, ${ }^{139}$ K. Sung, ${ }^{139}$ D. Velicanu, ${ }^{139}$ E. A. Wenger, ${ }^{139}$ R. Wolf, ${ }^{139}$ B. Wyslouch, ${ }^{139}$ S. Xie, ${ }^{139}$ M. Yang, ${ }^{139}$ Y. Yilmaz, ${ }^{139}$ A. S. Yoon, ${ }^{139}$ M. Zanetti, ${ }^{139}$ S. I. Cooper,${ }^{140}$ P. Cushman, ${ }^{140}$ B. Dahmes, ${ }^{140}$ A. De Benedetti, ${ }^{140}$ G. Franzoni, ${ }^{140}$ A. Gude, ${ }^{140}$ J. Haupt ${ }^{140}$ S. C. Kao, ${ }^{140}$ K. Klapoetke, ${ }^{140}$ Y. Kubota, ${ }^{140}$ J. Mans, ${ }^{140}$ N. Pastika, ${ }^{140}$ R. Rusack, ${ }^{140}$ M. Sasseville, ${ }^{140}$ A. Singovsky, ${ }^{140}$ N. Tambe, ${ }^{140}$ J. Turkewitz, ${ }^{140}$ L. M. Cremaldi, ${ }^{141}$ R. Kroeger,${ }^{141}$ L. Perera, ${ }^{141}$ R. Rahmat, ${ }^{141}$ D. A. Sanders, ${ }^{141}$ E. Avdeeva, ${ }^{142}$ K. Bloom, ${ }^{142}$ S. Bose,${ }^{142}$ J. Butt, ${ }^{142}$ D. R. Claes, ${ }^{142}$ A. Dominguez,${ }^{142}$ M. Eads, ${ }^{142}$ P. Jindal, ${ }^{142}$ J. Keller, ${ }^{142}$ I. Kravchenko, ${ }^{142}$ J. Lazo-Flores, ${ }^{142}$ H. Malbouisson, ${ }^{142}$ S. Malik, ${ }^{142}$ G. R. Snow, ${ }^{142}$ U. Baur, ${ }^{143}$ A. Godshalk, ${ }^{143}$ I. Iashvili, ${ }^{143}$ S. Jain, ${ }^{143}$ A. Kharchilava, ${ }^{143}$ A. Kumar,${ }^{143}$ S. P. Shipkowski, ${ }^{143}$ K. Smith,,${ }^{143}$ G. Alverson, ${ }^{144}$ E. Barberis, ${ }^{144}$ D. Baumgartel, ${ }^{144}$ M. Chasco, ${ }^{144}$ J. Haley, ${ }^{144}$ D. Nash, ${ }^{144}$ D. Trocino, ${ }^{144}$ D. Wood, ${ }^{144}$ J. Zhang, ${ }^{144}$ A. Anastassov, ${ }^{145}$ A. Kubik,,${ }^{145}$ N. Mucia, ${ }^{145}$ N. Odell, ${ }^{145}$ R. A. Ofierzynski, ${ }^{145}$ B. Pollack, ${ }^{145}$ A. Pozdnyakov, ${ }^{145}$ M. Schmitt, ${ }^{145}$ S. Stoynev, ${ }^{145}$ M. Velasco, ${ }^{145}$ S. Won, ${ }^{145}$ L. Antonelli, ${ }^{146}$ D. Berry, ${ }^{146}$ A. Brinkerhoff, ${ }^{146}$ M. Hildreth, ${ }^{146}$ C. Jessop, ${ }^{146}$ D. J. Karmgard, ${ }^{146}$ J. Kolb,${ }^{146}$ K. Lannon, ${ }^{146}$ W. Luo, ${ }^{146}$ S. Lynch, ${ }^{146}$ N. Marinelli, ${ }^{146}$ D. M. Morse, ${ }^{146}$ T. Pearson, ${ }^{146}$ R. Ruchti,${ }^{146}$ J. Slaunwhite, ${ }^{146}$ N. Valls, ${ }^{146}$ M. Wayne, ${ }^{146}$ M. Wolf, ${ }^{146}$ B. Bylsma, ${ }^{147}$ L. S. Durkin, ${ }^{147}$ A. Hart,${ }^{147}$ C. Hill,${ }^{147}$ R. Hughes,,${ }^{147}$ K. Kotov, ${ }^{147}$ T. Y. Ling,${ }^{147}$ D. Puigh,${ }^{147}$ M. Rodenburg, ${ }^{147}$ C. Vuosalo, ${ }^{147}$ G. Williams, ${ }^{147}$ B. L. Winer, ${ }^{147}$ N. Adam, ${ }^{148}$ E. Berry, ${ }^{148}$ P. Elmer ${ }^{148}$ D. Gerbaudo, ${ }^{148}$ V. Halyo, ${ }^{148}$ P. Hebda, ${ }^{148}$ J. Hegeman, ${ }^{148}$ A. Hunt, ${ }^{148}$ D. Lopes Pegna, ${ }^{148}$ P. Lujan, ${ }^{148}$ D. Marlow, ${ }^{148}$ T. Medvedeva, ${ }^{148}$ M. Mooney, ${ }^{148}$ J. Olsen, ${ }^{148}$ P. Piroué, ${ }^{148}$ X. Quan, ${ }^{148}$ A. Raval, ${ }^{148}$ H. Saka, ${ }^{148}$ D. Stickland ${ }^{148}$ C. Tully, ${ }^{148}$ J. S. Werner, ${ }^{148}$ A. Zuranski, ${ }^{148}$ J. G. Acosta, ${ }^{149}$ E. Brownson, ${ }^{149}$ X. T. Huang, ${ }^{149}$ A. Lopez, ${ }^{149}$ H. Mendez ${ }^{149}$ S. Oliveros, ${ }^{149}$ J.E. Ramirez Vargas, ${ }^{149}$ A. Zatserklyaniy, ${ }^{149}$ E. Alagoz, ${ }^{150}$ V.E. Barnes, ${ }^{150}$ D. Benedetti, ${ }^{150}$ G. Bolla, ${ }^{150}$ D. Bortoletto, ${ }^{150}$ M. De Mattia, ${ }^{150}$ A. Everett, ${ }^{150}$ Z. Hu, ${ }^{150}$ M. Jones, ${ }^{150}$ O. Koybasi,${ }^{150}$ M. Kress, ${ }^{150}$ A. T. Laasanen, ${ }^{150}$ N. Leonardo, ${ }^{150}$ V. Maroussov, ${ }^{150}$ P. Merkel, ${ }^{150}$ D. H. Miller, ${ }^{150}$ N. Neumeister,${ }^{150}$ I. Shipsey, ${ }^{150}$ D. Silvers, ${ }^{150}$ A. Svyatkovskiy, ${ }^{150}$ M. Vidal Marono, ${ }^{150}$ H. D. Yoo, ${ }^{150}$ J. Zablocki, ${ }^{150}$ Y. Zheng, ${ }^{150}$ S. Guragain, ${ }^{151}$ N. Parashar, ${ }^{151}$ A. Adair, ${ }^{152}$ C. Boulahouache, ${ }^{152}$ V. Cuplov, ${ }^{152}$ K. M. Ecklund, ${ }^{152}$ F. J. M. Geurts, ${ }^{152}$ B. P. Padley, ${ }^{152}$ R. Redjimi, ${ }^{152}$ J. Roberts, ${ }^{152}$ J. Zabel,${ }^{152}$ B. Betchart,${ }^{153}$ A. Bodek,${ }^{153}$ Y. S. Chung, ${ }^{153}$ R. Covarelli, ${ }^{153}$ 
P. de Barbaro, ${ }^{153}$ R. Demina, ${ }^{153}$ Y. Eshaq, ${ }^{153}$ A. Garcia-Bellido, ${ }^{153}$ P. Goldenzweig, ${ }^{153}$ Y. Gotra, ${ }^{153}$ J. Han, ${ }^{153}$ A. Harel,${ }^{153}$ S. Korjenevski, ${ }^{153}$ D. C. Miner ${ }^{153}$ D. Vishnevskiy, ${ }^{153}$ M. Zielinski, ${ }^{153}$ A. Bhatti, ${ }^{154}$ R. Ciesielski, ${ }^{154}$ L. Demortier, ${ }^{154}$ K. Goulianos, ${ }^{154}$ G. Lungu, ${ }^{154}$ S. Malik, ${ }^{154}$ C. Mesropian, ${ }^{154}$ S. Arora ${ }^{155}$ A. Barker, ${ }^{155}$ J. P. Chou, ${ }^{155}$ C. Contreras-Campana, ${ }^{155}$ E. Contreras-Campana, ${ }^{155}$ D. Duggan, ${ }^{155}$ D. Ferencek, ${ }^{155}$ Y. Gershtein,${ }^{155}$ R. Gray, ${ }^{155}$ E. Halkiadakis, ${ }^{155}$ D. Hidas, ${ }^{155}$ A. Lath,${ }^{155}$ S. Panwalkar, ${ }^{155}$ M. Park,${ }^{155}$ R. Patel,,${ }^{155}$ V. Rekovic, ${ }^{155}$ A. Richards, ${ }^{155}$ J. Robles,${ }^{155}$ K. Rose, ${ }^{155}$ S. Salur, ${ }^{155}$ S. Schnetzer, ${ }^{155}$ C. Seitz, ${ }^{155}$ S. Somalwar, ${ }^{155}$ R. Stone, ${ }^{155}$ S. Thomas, ${ }^{155}$ G. Cerizza, ${ }^{156}$ M. Hollingsworth, ${ }^{156}$ S. Spanier, ${ }^{156}$ Z. C. Yang, ${ }^{156}$ A. York,,${ }^{156}$ R. Eusebi, ${ }^{157}$ W. Flanagan, ${ }^{157}$ J. Gilmore, ${ }^{157}$ T. Kamon, ${ }^{157, \text { ddd }}$ V. Khotilovich, ${ }^{157}$ R. Montalvo, ${ }^{157}$ I. Osipenkov, ${ }^{157}$ Y. Pakhotin, ${ }^{157}$ A. Perloff, ${ }^{157}$ J. Roe, ${ }^{157}$ A. Safonov, ${ }^{157}$ T. Sakuma, ${ }^{157}$ S. Sengupta, ${ }^{157}$ I. Suarez, ${ }^{157}$ A. Tatarinov, ${ }^{157}$ D. Toback, ${ }^{157}$ N. Akchurin, ${ }^{158}$ J. Damgov, ${ }^{158}$ P. R. Dudero, ${ }^{158}$ C. Jeong, ${ }^{158}$ K. Kovitanggoon, ${ }^{158}$ S. W. Lee, ${ }^{158}$ T. Libeiro, ${ }^{158}$ Y. Roh, ${ }^{158}$ I. Volobouev, ${ }^{158}$ E. Appelt, ${ }^{159}$ D. Engh,${ }^{159}$ C. Florez, ${ }^{159}$ S. Greene, ${ }^{159}$ A. Gurrola, ${ }^{159}$ W. Johns,${ }^{159}$ C. Johnston, ${ }^{159}$ P. Kurt, ${ }^{159}$ C. Maguire, ${ }^{159}$ A. Melo, ${ }^{159}$ P. Sheldon, ${ }^{159}$ B. Snook,${ }^{159}$ S. Tuo, ${ }^{159}$ J. Velkovska, ${ }^{159}$ M. W. Arenton, ${ }^{160}$ M. Balazs, ${ }^{160}$ S. Boutle,${ }^{160}$ B. Cox,${ }^{160}$ B. Francis,${ }^{160}$ J. Goodell, ${ }^{160}$ R. Hirosky, ${ }^{160}$ A. Ledovskoy, ${ }^{160}$ C. Lin,${ }^{160}$ C. Neu, ${ }^{160}$ J. Wood ${ }^{160}$ R. Yohay, ${ }^{160}$ S. Gollapinni, ${ }^{161}$ R. Harr,${ }^{161}$ P.E. Karchin, ${ }^{161}$ C. Kottachchi Kankanamge Don, ${ }^{161}$ P. Lamichhane, ${ }^{161}$ A. Sakharov,${ }^{161}$ M. Anderson, ${ }^{162}$ M. Bachtis,${ }^{162}$ D. Belknap, ${ }^{162}$ L. Borrello, ${ }^{162}$ D. Carlsmith ${ }^{162}$ M. Cepeda, ${ }^{162}$ S. Dasu, ${ }^{162}$ L. Gray, ${ }^{162}$ K. S. Grogg,${ }^{162}$ M. Grothe, ${ }^{162}$ R. Hall-Wilton, ${ }^{162}$ M. Herndon, ${ }^{162}$ A. Hervé, ${ }^{162}$ P. Klabbers, ${ }^{162}$ J. Klukas, ${ }^{162}$ A. Lanaro, ${ }^{162}$ C. Lazaridis, ${ }^{162}$ J. Leonard, ${ }^{162}$ R. Loveless, ${ }^{162}$ A. Mohapatra, ${ }^{162}$ I. Ojalvo, ${ }^{162}$ F. Palmonari, ${ }^{162}$ G. A. Pierro, ${ }^{162}$ I. Ross, ${ }^{162}$ A. Savin, ${ }^{162}$ W. H. Smith, ${ }^{162}$ and J. Swanson ${ }^{162}$

\title{
(CMS Collaboration)
}

\author{
${ }^{1}$ Yerevan Physics Institute, Yerevan, Armenia \\ ${ }^{2}$ Institut für Hochenergiephysik der OeAW, Wien, Austria \\ ${ }^{3}$ National Centre for Particle and High Energy Physics, Minsk, Belarus \\ ${ }^{4}$ Universiteit Antwerpen, Antwerpen, Belgium \\ ${ }^{5}$ Vrije Universiteit Brussel, Brussel, Belgium \\ ${ }^{6}$ Université Libre de Bruxelles, Bruxelles, Belgium \\ ${ }^{7}$ Ghent University, Ghent, Belgium \\ ${ }^{8}$ Université Catholique de Louvain, Louvain-la-Neuve, Belgium \\ ${ }^{9}$ Université de Mons, Mons, Belgium \\ ${ }^{10}$ Centro Brasileiro de Pesquisas Fisicas, Rio de Janeiro, Brazil \\ ${ }^{11}$ Universidade do Estado do Rio de Janeiro, Rio de Janeiro, Brazil \\ ${ }^{12}$ Instituto de Fisica Teorica, Universidade Estadual Paulista, Sao Paulo, Brazil \\ ${ }^{13}$ Institute for Nuclear Research and Nuclear Energy, Sofia, Bulgaria \\ ${ }^{14}$ University of Sofia, Sofia, Bulgaria \\ ${ }^{15}$ Institute of High Energy Physics, Beijing, China \\ ${ }^{16}$ State Key Lab. of Nucl. Phys. and Tech., Peking University, Beijing, China \\ ${ }^{17}$ Universidad de Los Andes, Bogota, Colombia \\ ${ }^{18}$ Technical University of Split, Split, Croatia \\ ${ }^{19}$ University of Split, Split, Croatia \\ ${ }^{20}$ Institute Rudjer Boskovic, Zagreb, Croatia \\ ${ }^{21}$ University of Cyprus, Nicosia, Cyprus \\ ${ }^{22}$ Charles University, Prague, Czech Republic \\ ${ }^{23}$ Academy of Scientific Research and Technology of the Arab Republic of Egypt, \\ Egyptian Network of High Energy Physics, Cairo, Egypt \\ ${ }^{24}$ National Institute of Chemical Physics and Biophysics, Tallinn, Estonia \\ ${ }^{25}$ Department of Physics, University of Helsinki, Helsinki, Finland \\ ${ }^{26}$ Helsinki Institute of Physics, Helsinki, Finland \\ ${ }^{27}$ Lappeenranta University of Technology, Lappeenranta, Finland \\ ${ }^{28}$ DSM/IRFU, CEA/Saclay, Gif-sur-Yvette, France \\ ${ }^{29}$ Laboratoire Leprince-Ringuet, Ecole Polytechnique, IN2P3-CNRS, Palaiseau, France \\ ${ }^{30}$ Institut Pluridisciplinaire Hubert Curien, Université de Strasbourg, Université de Haute Alsace Mulhouse, \\ CNRS/IN2P3, Strasbourg, France \\ ${ }^{31}$ Centre de Calcul de l'Institut National de Physique Nucleaire et de Physique des Particules (IN2P3), Villeurbanne, France \\ ${ }^{32}$ Université de Lyon, Université Claude Bernard Lyon 1, CNRS-IN2P3, Institut de Physique Nucléaire de Lyon, Villeurbanne, France \\ ${ }^{33}$ Institute of High Energy Physics and Informatization, Tbilisi State University, Tbilisi, Georgia
}


${ }^{34}$ RWTH Aachen University, I. Physikalisches Institut, Aachen, Germany

${ }^{35}$ RWTH Aachen University, III. Physikalisches Institut A, Aachen, Germany

${ }^{36}$ RWTH Aachen University, III. Physikalisches Institut B, Aachen, Germany

${ }^{37}$ Deutsches Elektronen-Synchrotron, Hamburg, Germany

${ }^{38}$ University of Hamburg, Hamburg, Germany

${ }^{39}$ Institut für Experimentelle Kernphysik, Karlsruhe, Germany

${ }^{40}$ Institute of Nuclear Physics "Demokritos", Aghia Paraskevi, Greece

${ }^{41}$ University of Athens, Athens, Greece

${ }^{42}$ University of Ioánnina, Ioánnina, Greece

${ }^{43}$ KFKI Research Institute for Particle and Nuclear Physics, Budapest, Hungary

${ }^{44}$ Institute of Nuclear Research ATOMKI, Debrecen, Hungary

${ }^{45}$ University of Debrecen, Debrecen, Hungary

${ }^{46}$ Panjab University, Chandigarh, India

${ }^{47}$ University of Delhi, Delhi, India

${ }^{48}$ Saha Institute of Nuclear Physics, Kolkata, India

${ }^{49}$ Bhabha Atomic Research Centre, Mumbai, India

${ }^{50}$ Tata Institute of Fundamental Research-EHEP, Mumbai, India

${ }^{51}$ Tata Institute of Fundamental Research-HECR, Mumbai, India

${ }^{52}$ Institute for Research in Fundamental Sciences (IPM), Tehran, Iran

${ }^{53 a}$ INFN Sezione di Bari, Italy

${ }^{53 \mathrm{~b}}$ Università di Bari, Italy

${ }^{53 \mathrm{c}}$ Politecnico di Bari, Italy

${ }^{54 a}$ INFN Sezione di Bologna, Bologna, Italy

${ }^{54 b}$ Università di Bologna, Bologna, Italy

${ }^{55 a}$ INFN Sezione di Catania, Catania, Italy

${ }^{55 \mathrm{~b}}$ Università di Catania, Catania, Italy

${ }^{56 a}$ INFN Sezione di Firenze, Firenze, Italy

${ }^{56 \mathrm{~b}}$ Università di Firenze, Firenze, Italy

${ }^{57}$ INFN Laboratori Nazionali di Frascati, Frascati, Italy

${ }^{58}$ INFN Sezione di Genova, Genova, Italy

${ }^{59 a}$ INFN Sezione di Milano-Bicocca, Milano, Italy

${ }^{59 \mathrm{~b}}$ Università di Milano-Bicocca, Milano, Italy

${ }^{60 a}$ INFN Sezione di Napoli, Napoli, Italy

${ }^{60 \mathrm{~b}}$ Università di Napoli "Federico II", Napoli, Italy

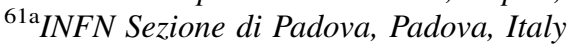

${ }^{61 \mathrm{~b}}$ Università di Padova, Padova, Italy

${ }^{61 \mathrm{c}}$ Università di Trento (Trento), Padova, Italy

${ }^{62 a}$ INFN Sezione di Pavia, Pavia, Italy

${ }^{62 \mathrm{~b}}$ Università di Pavia, Pavia, Italy

${ }^{63 a}$ INFN Sezione di Perugia, Perugia, Italy

${ }^{63 \mathrm{~b}}$ Università di Perugia, Perugia, Italy

${ }^{64 a}$ INFN Sezione di Pisa, Pisa, Italy

${ }^{64 \mathrm{~b}}$ Università di Pisa, Pisa, Italy

${ }^{64 \mathrm{c}}$ Scuola Normale Superiore di Pisa, Pisa, Italy

${ }^{65}$ aNFN Sezione di Roma, Roma, Italy

${ }^{65 b}$ Università di Roma "La Sapienza”, Roma, Italy

${ }^{66 a}$ INFN Sezione di Torino, Torino, Italy

${ }^{66 \mathrm{~b}}$ Università di Torino, Torino, Italy

${ }^{66 c}$ Università del Piemonte Orientale (Novara), Torino, Italy

${ }^{67 a}$ INFN Sezione di Trieste, Trieste, Italy

${ }^{67 \mathrm{~b}}$ Università di Trieste, Trieste, Italy

${ }^{68}$ Kangwon National University, Chunchon, Korea

${ }^{69}$ Kyungpook National University, Daegu, Korea

${ }^{70}$ Chonnam National University, Institute for Universe and Elementary Particles, Kwangju, Korea

${ }^{71}$ Konkuk University, Seoul, Korea

${ }^{72}$ Korea University, Seoul, Korea

${ }^{73}$ University of Seoul, Seoul, Korea

${ }^{74}$ Sungkyunkwan University, Suwon, Korea

${ }^{75}$ Vilnius University, Vilnius, Lithuania

${ }^{76}$ Centro de Investigacion y de Estudios Avanzados del IPN, Mexico City, Mexico

${ }^{77}$ Universidad Iberoamericana, Mexico City, Mexico 
${ }^{78}$ Benemerita Universidad Autonoma de Puebla, Puebla, Mexico

${ }^{79}$ Universidad Autónoma de San Luis Potosí, San Luis Potosí, Mexico

${ }^{80}$ University of Auckland, Auckland, New Zealand

${ }^{81}$ University of Canterbury, Christchurch, New Zealand

${ }^{82}$ National Centre for Physics, Quaid-I-Azam University, Islamabad, Pakistan

${ }^{83}$ Institute of Experimental Physics, Faculty of Physics, University of Warsaw, Warsaw, Poland

${ }^{84}$ Soltan Institute for Nuclear Studies, Warsaw, Poland

${ }^{85}$ Laboratório de Instrumentação e Física Experimental de Partículas, Lisboa, Portugal

${ }^{86}$ Joint Institute for Nuclear Research, Dubna, Russia

${ }^{87}$ Petersburg Nuclear Physics Institute, Gatchina (St Petersburg), Russia

${ }^{88}$ Institute for Nuclear Research, Moscow, Russia

${ }^{89}$ Institute for Theoretical and Experimental Physics, Moscow, Russia

${ }^{90}$ Moscow State University, Moscow, Russia

${ }^{91}$ P. N. Lebedev Physical Institute, Moscow, Russia

${ }^{92}$ State Research Center of Russian Federation, Institute for High Energy Physics, Protvino, Russia

${ }^{93}$ University of Belgrade, Faculty of Physics and Vinca Institute of Nuclear Sciences, Belgrade, Serbia

${ }^{94}$ Centro de Investigaciones Energéticas Medioambientales y Tecnológicas (CIEMAT), Madrid, Spain

${ }^{95}$ Universidad Autónoma de Madrid, Madrid, Spain

${ }^{96}$ Universidad de Oviedo, Oviedo, Spain

${ }^{97}$ Instituto de Física de Cantabria (IFCA), CSIC-Universidad de Cantabria, Santander, Spain

${ }^{98}$ CERN, European Organization for Nuclear Research, Geneva, Switzerland

${ }^{99}$ Paul Scherrer Institut, Villigen, Switzerland

${ }^{100}$ Institute for Particle Physics, ETH Zurich, Zurich, Switzerland

${ }^{101}$ Universität Zürich, Zurich, Switzerland

${ }^{102}$ National Central University, Chung-Li, Taiwan

${ }^{103}$ National Taiwan University (NTU), Taipei, Taiwan

${ }^{104}$ Cukurova University, Adana, Turkey

${ }^{105}$ Middle East Technical University, Physics Department, Ankara, Turkey

${ }^{106}$ Bogazici University, Istanbul, Turkey

${ }^{107}$ Istanbul Technical University, Istanbul, Turkey

${ }^{108}$ National Scientific Center, Kharkov Institute of Physics and Technology, Kharkov, Ukraine

${ }^{109}$ University of Bristol, Bristol, United Kingdom

${ }^{110}$ Rutherford Appleton Laboratory, Didcot, United Kingdom

${ }^{111}$ Imperial College, London, United Kingdom

${ }^{112}$ Brunel University, Uxbridge, United Kingdom

${ }^{113}$ Baylor University, Waco, Texas, USA

${ }^{114}$ The University of Alabama, Tuscaloosa, Alabama, USA

${ }^{115}$ Boston University, Boston, Massachusetts, USA

${ }^{116}$ Brown University, Providence, Rhode Island, USA

${ }^{117}$ University of California, Davis, Davis, California, USA

${ }^{118}$ University of California, Los Angeles, Los Angeles, California, USA

${ }^{119}$ University of California, Riverside, Riverside, California, USA

${ }^{120}$ University of California, San Diego, La Jolla, California, USA

${ }^{121}$ University of California, Santa Barbara, Santa Barbara, California, USA

${ }^{122}$ California Institute of Technology, Pasadena, California, USA

${ }^{123}$ Carnegie Mellon University, Pittsburgh, Pennsylvania, USA

${ }^{124}$ University of Colorado at Boulder, Boulder, Colorado, USA

${ }^{125}$ Cornell University, Ithaca, New York USA

${ }^{126}$ Fairfield University, Fairfield, Connecticut, USA

${ }^{127}$ Fermi National Accelerator Laboratory, Batavia, Illinois, USA

${ }^{128}$ University of Florida, Gainesville, Florida, USA

${ }^{129}$ Florida International University, Miami, Florida, USA

${ }^{130}$ Florida State University, Tallahassee, Florida, USA

${ }^{131}$ Florida Institute of Technology, Melbourne, Florida, USA

${ }^{132}$ University of Illinois at Chicago (UIC), Chicago, Illinois, USA

${ }^{133}$ The University of Iowa, Iowa City, Iowa, USA

${ }^{134}$ Johns Hopkins University, Baltimore, Maryland, USA

${ }^{135}$ The University of Kansas, Lawrence, Kansas, USA

${ }^{136}$ Kansas State University, Manhattan, Kansas, USA

${ }^{137}$ Lawrence Livermore National Laboratory, Livermore, California, USA

${ }^{138}$ University of Maryland, College Park, Maryland, USA 
${ }^{139}$ Massachusetts Institute of Technology, Cambridge, Massachusetts, USA

${ }^{140}$ University of Minnesota, Minneapolis, Minnesota, USA

${ }^{141}$ University of Mississippi, University, Mississippi, USA

${ }^{142}$ University of Nebraska-Lincoln, Lincoln, Nebraska, USA

${ }^{143}$ State University of New York at Buffalo, Buffalo, New York, USA

${ }^{144}$ Northeastern University, Boston, Massachusetts, USA

${ }^{145}$ Northwestern University, Evanston, Illinois, USA

${ }^{146}$ University of Notre Dame, Notre Dame, Indiana, USA

${ }^{147}$ The Ohio State University, Columbus, Ohio, USA

${ }^{148}$ Princeton University, Princeton, New Jersey, USA

${ }^{149}$ University of Puerto Rico, Mayaguez, USA

${ }^{150}$ Purdue University, West Lafayette, Indiana, USA

${ }^{151}$ Purdue University Calumet, Hammond, Indiana, USA

${ }^{152}$ Rice University, Houston, Texas, USA

${ }^{153}$ University of Rochester, Rochester, New York, USA

${ }^{154}$ The Rockefeller University, New York, New York, USA

${ }^{155}$ Rutgers, The State University of New Jersey, Piscataway, New York, USA

${ }^{156}$ University of Tennessee, Knoxville, Tennessee, USA

${ }^{157}$ Texas A\&M University, College Station, Texas, USA

${ }^{158}$ Texas Tech University, Lubbock, Texas, USA

${ }^{159}$ Vanderbilt University, Nashville, Tennessee, USA

${ }^{160}$ University of Virginia, Charlottesville, Virginia, USA

${ }^{161}$ Wayne State University, Detroit, Michigan, USA

${ }^{162}$ University of Wisconsin, Madison, Wisconsin, USA

${ }^{\mathrm{a}}$ Deceased.

${ }^{\mathrm{b}}$ Also at Vienna University of Technology, Vienna, Austria.

${ }^{\mathrm{c}}$ Also at National Institute of Chemical Physics and Biophysics, Tallinn, Estonia.

${ }^{\mathrm{d}}$ Also at Universidade Federal do ABC, Snto Ndre, Brazil.

${ }^{\mathrm{e}}$ Also at California Institute of Technology, Pasadena, USA.

${ }_{\mathrm{f}}^{\mathrm{f}}$ Also at CERN, Europen Orgnization for Nuclear Research, Geneva, Switzerland.

${ }^{\mathrm{g}}$ Also at Laboratoire Leprince-Ringuet, Ecole Polytechnique, IN2P3-CNRS, Palaiseau, France.

${ }^{\mathrm{h}}$ Also at Suez Canal University, Suez, Egypt.

${ }^{\mathrm{i}}$ Also at Zewail City of Science nd Technology, Zewail, Egypt.

${ }^{\mathrm{j}}$ Also at Cairo University, Cairo, Egypt.

${ }^{\mathrm{k}}$ Also at Fayoum University, El-Fayoum, Egypt.

${ }^{1}$ Also at Ain Shams University, Cairo, Egypt.

${ }^{\mathrm{m}}$ Also at Soltn Institute for Nuclear Studies, Warsaw, Poland.

${ }^{\text {n}}$ Also at Université de Haute-Alsace, Mulhouse, France.

${ }^{\circ}$ Also at Moscow State University, Moscow, Russia.

${ }^{\mathrm{p}}$ Also at Brandenburg University of Technology, Cottbus, Germany.

${ }^{\mathrm{q}}$ Also at Institute of Nuclear Research ATOMKI, Debrecen, Hungary.

${ }^{r}$ Also at Eötvös Loránd University, Budapest, Hungary.

${ }^{\mathrm{s}}$ Also at Tata Institute of Fundamental Research - HECR, Mumbai, India.

${ }^{\mathrm{t}}$ Also at University of Visva-Bharati, Santiniketan, India.

uAlso at Sharif University of Technology, Tehran, Iran.

${ }^{\mathrm{v}}$ Also at Isfahan University of Technology, Isfahan, Iran.

${ }^{\mathrm{w}}$ Also at Shiraz University, Shiraz, Iran.

${ }^{\mathrm{x}}$ Also at Plasma Physics Research Center, Science and Research Branch, Islamic Azad University, Teheran, Iran.

${ }^{\mathrm{y}}$ Also at Facoltà Ingegneria Università di Roma, Roma, Italy.

${ }^{\mathrm{z}}$ Also at Università della Basilicata, Potenza, Italy.

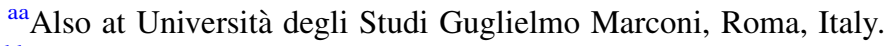

${ }^{\mathrm{bb}}$ Also at Università degli studi di Siena, Siena, Italy.

${ }^{\mathrm{cc}}$ Also at University of Bucharest, Faculty of Physics, Bucuresti-Magurele, Romania.

${ }^{\mathrm{dd}}$ Also at Faculty of Physics of University of Belgrade, Belgrade, Serbia.

${ }^{e e}$ Also at University of Florida, Gainesville, USA.

${ }^{\mathrm{ff}}$ Also at University of California, Los Angeles, Los Angeles, USA. 
${ }^{\text {gg }}$ Also at Scuola Normale e Sezione dell' INFN, Pisa, Italy.

hh Also at INFN Sezione di Roma, Università di Roma "La Sapienza", Roma, Italy.

${ }^{\mathrm{ii}}$ Also at University of Athens, Athens, Greece.

${ }^{\mathrm{jj}}$ Also at Rutherford Appleton Laboratory, Didcot, United Kingdom.

${ }^{\mathrm{kk}}$ Also at The University of Kansas, Lawrence, USA.

${ }^{11}$ Also at Paul Scherrer Institut, Villigen, Switzerland.

${ }^{\mathrm{mm}}$ Also at Institute for Theoretical and Experimental Physics, Moscow, Russia.

${ }^{n n}$ Also at Gaziosmanpasa University, Tokat, Turkey.

${ }^{\circ o}$ Also at Adiyaman University, Adiyaman, Turkey.

${ }^{\mathrm{pp}}$ Also at The University of Iowa, Iowa City, USA.

${ }^{\mathrm{qq}}$ Also at Mersin University, Mersin, Turkey.

${ }^{\mathrm{rr}}$ Also at Ozyegin University, Istanbul, Turkey.

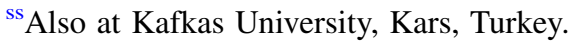

${ }^{\text {tt} A l s o ~ a t ~ S u l e y m a n ~ D e m i r e l ~ U n i v e r s i t y, ~ I s p a r t a, ~ T u r k e y . ~}$

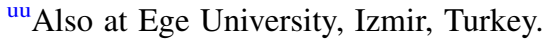

${ }^{v v}$ Also at School of Physics and Astronomy, University of Southampton, Southampton, United Kingdom.

${ }^{w w}$ Also at INFN Sezione di Perugia, Università di Perugia, Perugia, Italy.

${ }^{\mathrm{xx}}$ Also at University of Sydney, Sydney, Australia.

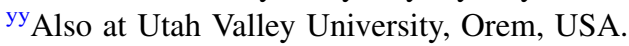

${ }^{\mathrm{zz}}$ Also at Institute for Nuclear Research, Moscow, Russia.

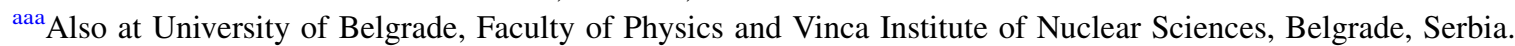

${ }^{\mathrm{bbb}}$ Also at Argonne National Laboratory, Argonne, USA.

${ }^{\mathrm{ccc}}$ Also at Erzincan University, Erzincan, Turkey.

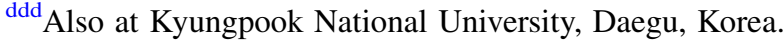

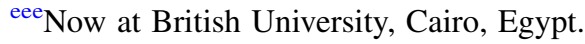

